This article has been accepted for publication in Monthly Notices of the Royal Astronomical Society (C): 2019 The Authors. Published by Oxford University Press on behalf of the Royal Astronomical Society. All rights reserved. 


\title{
Rotation measure synthesis applied to synthetic SKA images of galaxy clusters
}

\author{
F. Loi ${ }^{\oplus},{ }^{1,2 \star}$ M. Murgia ${ }^{\oplus},{ }^{2}$ F. Govoni, ${ }^{2}$ V. Vacca,${ }^{2}$ A. Bonafede, ${ }^{1}$ C. Ferrari, ${ }^{3}$ \\ I. Prandoni ${ }^{\oplus},{ }^{4}$ L. Feretti, ${ }^{4}$ G. Giovannini ${ }^{1}$ and $\mathrm{H} . \mathrm{Li}^{5}$ \\ ${ }^{1}$ Dip. di Fisica e Astronomia, Università degli Studi Bologna, Viale Berti Pichat 6/2, I-40127 Bologna, Italy \\ ${ }^{2}$ INAF - Osservatorio Astronomico di Cagliari, Via della Scienza 5, I-09047 Selargius (CA), Italy \\ ${ }^{3}$ Observatoire de la Cote d'Azur, France \\ ${ }^{4}$ INAF - Istituto di Radioastronomia, Via Gobetti 101, I-40129 Bologna, Italy \\ ${ }^{5}$ Theoretical Astrophysics, Los Alamos National Laboratory, Los Alamos, NM, USA
}

Accepted 2019 September 16. Received 2019 September 6; in original form 2019 July 15

\begin{abstract}
Future observations with next-generation radio telescopes will help us to understand the presence and evolution of magnetic fields in galaxy clusters through determination of the socalled rotation measure (RM). In this work, we applied the RM synthesis technique to synthetic first phase Square Kilometre Array mid frequency element (i.e. the SKA1-MID) radio images of a pair of merging galaxy clusters, measured between 950 and $1750 \mathrm{MHz}$ with a resolution of $10 \mathrm{arcsec}$ and thermal noise of $0.1 \mu \mathrm{Jy}$ beam $^{-1}$. The results of our RM synthesis analysis are compared with the simulation input parameters. We study two cases: one with radio haloes at the cluster centres and another without. We found that the information obtained with RM synthesis is in general agreement with the input information; however, some discrepancies are present. We characterize them in this work, with the final goal of determining the potential impact of SKA1-MID on the study of cluster magnetic fields.
\end{abstract}

Key words: polarization-methods: numerical-galaxies: clusters: intracluster mediummagnetic fields.

\section{INTRODUCTION}

The next generation of radio instruments is allowing us to investigate cosmic magnetism with unprecedented insight. Indeed, our Universe is highly magnetized on all scales, from the smallest objects (stars and planets) to the largest (galaxies and galaxy clusters). Hints of the presence of magnetic fields are observed even on larger scales, namely in the filaments of the cosmic web (Vacca et al. 2018; Govoni et al. 2019).

Current models predict that magnetic fields in galaxy clusters could originate either from a primordial injection or from seeding from galactic outflows: the injected magnetic field would then be amplified and spread all over the intracluster medium (ICM) via turbulence and shock waves, up to a strength of few $\mu \mathrm{G}$ on Mpc scales (Donnert et al. 2018). In order to distinguish between different scenarios, it is important to study the magnetic fields in galaxy clusters and filaments through both numerical simulations and observations.

The effect of Faraday rotation on background and cluster radio sources (e.g. Clarke 2004; Govoni \& Feretti 2004) is very useful to determine the intracluster magnetic field properties. The Faraday

^E-mail: francesca.loi@inaf.it effect manifests as the rotation of the polarization plane of a linearly polarized signal passing through a magnetoionic medium and depends on a function of the square of the wavelength, $\lambda^{2}$, and the Faraday depth, $\phi(l)$, as follows:

$\Delta \Psi=\Psi-\Psi_{0}=\phi(l) \lambda^{2}$.

The Faraday depth is the integral of the line-of-sight parallel component of the intervening magnetic field multiplied by the thermal plasma density:

$\phi(l)=\int_{0}^{l} B_{\|} n_{\mathrm{e}} \mathrm{d} l$,

where the integral is performed over the path crossed by the signal. In the case of a radio source with signal passing through the magnetoionic medium of a galaxy cluster and observed polarized emission following the $\lambda^{2}$ dependence of equation (1), the associated Faraday depth is known as the cluster's rotation measure (RM) and the information it encodes is linked to the properties of that cluster, particularly its intracluster magnetic field. Evaluating the Faraday depth is useful to investigate the magnetic field on small scales $(\sim \mathrm{kpc})$, but its exploitation is limited by the current sensitivity of radio telescopes, which does not allow us to detect more than a few polarized radio sources per cluster and only in a few clusters. 
Indeed, the densest RM grid at $1.4 \mathrm{GHz}$ available today has an average density of 1 RM source per square degree (Taylor, Stil \& Sunstrum 2009) and it is based on the National Radio Astronomy Observatory (NRAO) Very Large Array (VLA) Sky Survey (NVSS: Condon et al. 1998).

Some galaxy clusters show central diffuse radio emission, which is not associated with specific optical counterparts or discrete radio sources. The observed properties of these so-called radio haloes (see the reviews of Ferrari et al. 2008; Feretti et al. 2012; van Weeren et al. 2019) reveal their synchrotron nature and prove the existence of a non-thermal component in the ICM, made of relativistic particles and large-scale magnetic fields. Radio haloes are found at the centre of merging clusters and are typically $\sim 1 \mathrm{Mpc}$ in size. They are characterized by a low surface brightness $\left(S_{v} \sim 0.1-1 \mu \mathrm{Jy} \operatorname{arcsec}^{-2}\right.$ at $1.4 \mathrm{GHz})$, with a steep power-law spectrum $\left(S_{v}=S_{0}\left(v / v_{0}\right)^{-\alpha}\right.$, $\alpha \gtrsim 1)$. Although radio haloes are synchrotron sources, they are generally observed to be unpolarized, though polarized radio halo emission has been detected (up to $\sim 20$ percent at $1.4 \mathrm{GHz}$ ) in galaxy clusters A2255 (Govoni et al. 2005), MACS J0717.5 + 3745 (Bonafede et al. 2009) and A523 (Girardi et al. 2016). The absence of polarized emission in radio haloes could be attributed to two types of depolarization effects: internal and external depolarization. External depolarization is caused by instrumental effects. It occurs when the emitting magnetic field is tangled on a scale smaller than the beam area of the telescope and can also be observed in the case of discrete radio sources. In addition, Faraday rotation can take place within a frequency channel and the larger the channel, the larger the rotation. The result is an incoherent sum of polarized signals that, averaged together, effectively suppress the measured degree of polarization. Internal depolarization takes place whenever a radio-emitting source is mixed with a magnetoionic medium: every polarized signal emitted in a different position along a given line of sight will experience a different Faraday depth, corresponding to the crossed portion of the plasma; thus, all the signals coming from that line of sight will be rotated by different angles and when they reach the telescope they sum up incoherently in each frequency channel. As a result, the degree of polarization is strongly reduced at long wavelengths with respect to the intrinsic value that would be observed at $\lambda=0$.

To mitigate the impact of the problems mentioned above, it is common to use the RM synthesis technique (Burn 1966; Brentjens \& de Bruyn 2005). This consists of building a spectrum of the polarization as a function of the Faraday depth, known as the Faraday dispersion function $F(\phi)$. This spectrum is built for each given line of sight using the $Q$ and $U$ Stokes parameters measured by an instrument. Assuming a value of Faraday depth $\phi(l)^{*}$ in a given interval, $Q$ and $U$ are derotated in each frequency channel according to this value. The polarized intensity associated with this Faraday depth is then computed as

$\|P\|=\sqrt{Q^{2}+U^{2}}$,

where $P=Q+\mathrm{i} U$ and $Q$ and $U$ are real quantities. By repeating this procedure for different values of Faraday depths inside a given interval, it is possible to obtain an approximate reconstruction of the Faraday dispersion function. More formally, it can be shown that a Fourier relation exists between the polarization distribution in Faraday space and the observed polarization in the frequency domain. If this technique is applied to a background radio galaxy, in the absence of any Faraday screen except the cluster ICM itself, the result is a Faraday dispersion function with a single peak at the Faraday depth, which corresponds to the cluster RM. In the case of radio haloes, emitting sources mixed with rotating plasma or multiple sources along the line of sight, there will be several polarized peaks emitted at different positions along the path towards the telescope, which will experience different Faraday depths. The Faraday dispersion function can thus feature multiple components and determining which is associated with the cluster we are interested in can be a non-trivial task.

In this work, the RM synthesis is applied to synthetic radio images of galaxy clusters. These are the results of a cosmological magnetohydrodynamical (MHD) simulation of the ICM properties of a pair of merging galaxy clusters in combination with the FARADAY software package (Murgia et al. 2004). In previous works, this tool has been shown to be capable of reproducing the radio emission associated with a discrete radio source population (Loi et al. 2019) and cluster radio haloes (Govoni et al. 2013; $\mathrm{Xu}$ et al. 2012). Here, we focus on investigating the potential benefits of the RM synthesis technique applied to synthetic first phase Square Kilometre Array mid frequency element (i.e. the SKA1-MID) radio images, aiming to determine the cluster RM. Throughout the article, the following nomenclature is adopted: simulated images are images at the maximum resolution offered by the MHD simulations, i.e. $\sim 10.7 \mathrm{kpc}$ (which corresponds to $\sim 5$ arcsec at the cluster redshift $z=0.115$ ). The thermal noise in our simulated images is zero; synthetic images are the simulated images convolved with a Gaussian function having full width at half-maximum $(\mathrm{FWHM})=10$ arcsec after adding a thermal noise of $0.1 \mu \mathrm{Jybeam}^{-1}$. Intrinsic parameters refer to those physical observables that can be computed from the MHD cubes, such as the cluster RM. The simulated and synthetic images are produced considering the frequency band $950-1760 \mathrm{MHz}$ (i.e. band 2 of SKA1-MID). We treat two separate cases: clusters with radio haloes and clusters without radio haloes. The results of the RM synthesis applied to the data are compared with the intrinsic cluster RM.

The article is organized as follows: in Section 2, we describe the assumptions made to produce the simulated full Stokes images of the two merging clusters; in Section 3, we show the simulated and synthetic total intensity radio images. Section 4 describes the application of the RM-synthesis technique to simulated and synthetic data, along with how the results will be treated. Section 5 reports the results of the RM synthesis on simulated and synthetic images. Finally, Section 6 is devoted to analysis and Section 7 to our conclusions. In the Appendix, a simple estimate of the intracluster magnetic field strength is given.

Throughout the article, a $\Lambda \mathrm{CDM}$ cosmology is adopted with $H_{0}=71 \mathrm{~km} \mathrm{~s}^{-1} \mathrm{Mpc}^{-1}, \Omega_{\mathrm{m}}=0.27$ and $\Omega_{\Lambda}=0.73$. The galaxy clusters of this work are simulated at redshift $z=0.115$, where $1 \operatorname{arcsec}=2.06 \mathrm{kpc}$.

\section{CHARACTERISTICS OF THE SIMULATIONS}

In this section, we describe the models and simulations adopted to produce full Stokes spectropolarimetric cubes of the merging system. Cluster ICM properties (i.e. thermal density, temperature and magnetic field) are derived from a cosmological MHD simulation, while the total and polarized radio emission are simulated using previous properties.

\subsection{MHD simulations}

The cosmological MHD simulation presented here is obtained with the ENZO code (Collins et al. 2010) with adaptive mesh refinement (AMR) developed by the group of Hui Li at the Los Alamos National 
Table 1. Parameters of the MHD simulation: from left to right, the field of view (FOV), pixel size, mass and $r_{200}$ of cluster $\mathrm{C} 1$ (first row) and $\mathrm{C} 2$ (second row) are listed. The $\beta$-model parameters, namely the central density $n_{0}$, the core radius $r_{\mathrm{c}}$ and $\beta$, are also listed in this table.

\begin{tabular}{lcccccc}
\hline $\begin{array}{l}\text { FOV } \\
{[\mathrm{Mpc}]}\end{array}$ & $\begin{array}{c}\text { pix. size } \\
{[\mathrm{kpc}]}\end{array}$ & $\begin{array}{c}\text { mass } \\
{\left[10^{14} \mathrm{M}_{\odot}\right]}\end{array}$ & $\begin{array}{c}r_{200} \\
{[\mathrm{Mpc}]}\end{array}$ & $\begin{array}{c}n_{0} \\
{\left[\mathrm{~cm}^{-3}\right]}\end{array}$ & $\begin{array}{c}r_{\mathrm{c}} \\
{[\mathrm{kpc}]}\end{array}$ & $\beta$ \\
\hline $6.4^{2}$ & 10.7 & 4.7 & 1.2 & 0.0062 & 246.1 & 0.69 \\
& & 3.8 & 1.1 & 0.0067 & 192.6 & 0.63 \\
\hline
\end{tabular}

Laboratories, USA. It is a small part of a bigger cosmological simulation realized in a volume of $\left(256 h^{-1} \mathrm{Mpc}^{-3}\right)$ which encloses several forming structures. The evolution of dark matter, baryonic matter and magnetic fields is built into the simulation. An adiabatic equation of state with a specific heat ratio $\Gamma=5 / 3$ is used, while heating and cooling physics or chemical reactions are not included. The simulation runs from redshift $z=30$ to $z=0$. For this work, we focus on a pair of galaxy clusters with similar masses that undergoes a merging process, resulting in a single system at $z=0.073$, with a total mass $M=1.9 \times 10^{15} \mathrm{M}_{\odot}$. The process lasts for about $2 \mathrm{Gyr}$. The magnetic fields are injected by active galactic nuclei (AGNs) at $z=2-3$ (Xu et al. 2012) and then amplified and spread over Mpc scales during the late stages of the merger.

For the purposes of this work, a single snapshot of the MHD simulation at $z=0.115$ is used. At this particular instant, the cluster centres are separated by about $2 \mathrm{Mpc}$. The simulation consists of a set of three-dimensional cubes of $\sim(6.4 \mathrm{Mpc})^{3}$ with a cell size of $\sim 10.7 \mathrm{kpc}$ containing the ICM physical parameters: temperature, thermal plasma density and intracluster magnetic fields. The physical configuration is very similar to that observed for the pair of galaxy clusters A399-A401, where both the systems host a diffuse radio halo (Murgia et al. 2010).

The characteristics of the simulation are summarized in Table 1, where we also specified the $r_{200}$ radius, defined as the distance within which the cluster density is 200 times the critical density of the Universe.

\subsubsection{Temperature, density and $X$-ray emission}

Fig. 1 shows the temperature (left) and density (middle) profiles as a function of the distance from the cluster centres, computed from the three-dimensional MHD cubes, for the $\mathrm{C} 1$ (red) and $\mathrm{C} 2$ (blue) clusters. The simulated ICM reaches temperatures as high as $\sim 8-9 \times 10^{7} \mathrm{~K}$ in the cluster centre, decreasing towards the outskirts of the cluster for both $\mathrm{C} 1$ and $\mathrm{C} 2$, even if a clear enhancement can be observed in $\mathrm{C} 1$ at $\sim 700 \mathrm{kpc}$ from the cluster centre. At the cluster centres, the density assumes values of the order of $\sim$ $6 \times 10^{-3} \mathrm{~cm}^{-3}$, decreasing going to large distances. Even if this is a merging system, the density profiles are well described by a $\beta$ model (Cavaliere \& Fusco-Femiano 1976):

$n(r)=n_{0} \cdot\left(1+\left(\frac{r}{r_{c}}\right)^{2}\right)^{-3 \beta / 2}$,

with best-fitting parameters for the central density $n_{0}=$ $0.0062 \mathrm{~cm}^{-3}$, core radius $r_{\mathrm{c}}=246.1 \mathrm{kpc}$ and $\beta=0.69$ for the $\mathrm{C} 1$ cluster and $n_{0}=0.0067 \mathrm{~cm}^{-3}, r_{\mathrm{c}}=192.6 \mathrm{kpc}$ and $\beta=0.63$ for the $\mathrm{C} 2$ cluster. These parameters are reported in Table 1 . The best-fitting equations are plotted as dashed lines.

From the MHD cubes, it is possible to evaluate the X-ray surface brightness of the clusters in the energy band $0.1-2.4 \mathrm{keV}$. This is shown on the right of Fig. 1.

\subsubsection{Magnetic field and RM properties}

Fig. 2 shows on the left the average of the magnetic field profile of the $\mathrm{C} 1$ (red) and $\mathrm{C} 2$ (blue) clusters. Their intensities range from 0.1 to $1 \mu \mathrm{G}$. The $\mathrm{C} 1$ cluster magnetic field shows an enhancement of its intensity from $\sim 0.2$ up to $\sim 0.7 \mu \mathrm{G}$ in the range between 10 and $100 \mathrm{kpc}$ and then a decrease at distances larger than $\sim 500 \mathrm{kpc}$. The $\mathrm{C} 2$ cluster has a flat behaviour, with an intensity of $\sim 0.6-0.7 \mu \mathrm{G}$ up to $500 \mathrm{kpc}$ and then a decrease similar to the one observed in the C1 cluster.

Fig. 2 also shows, on the right, the magnetic field power spectra of the two clusters, computed as the Fourier transform of the 3D magnetic field autocorrelation function in the wavenumber domain $k=2 \pi / \Lambda$, where $\Lambda$ is the scale in $\mathrm{kpc}$. As before, the power spectra of the $\mathrm{C} 1$ and $\mathrm{C} 2$ clusters are traced in red and blue, respectively. For both the clusters, the magnetic field autocorrelation lengths have been evaluated from (Enßlin \& Vogt 2003)

$\Lambda_{B}=\frac{3 \pi}{2} \frac{\int_{0}^{\infty}\left|B_{k}\right|^{2} k \mathrm{~d} k}{\int_{0}^{\infty}\left|B_{k}\right|^{2} k^{2} \mathrm{~d} k}$
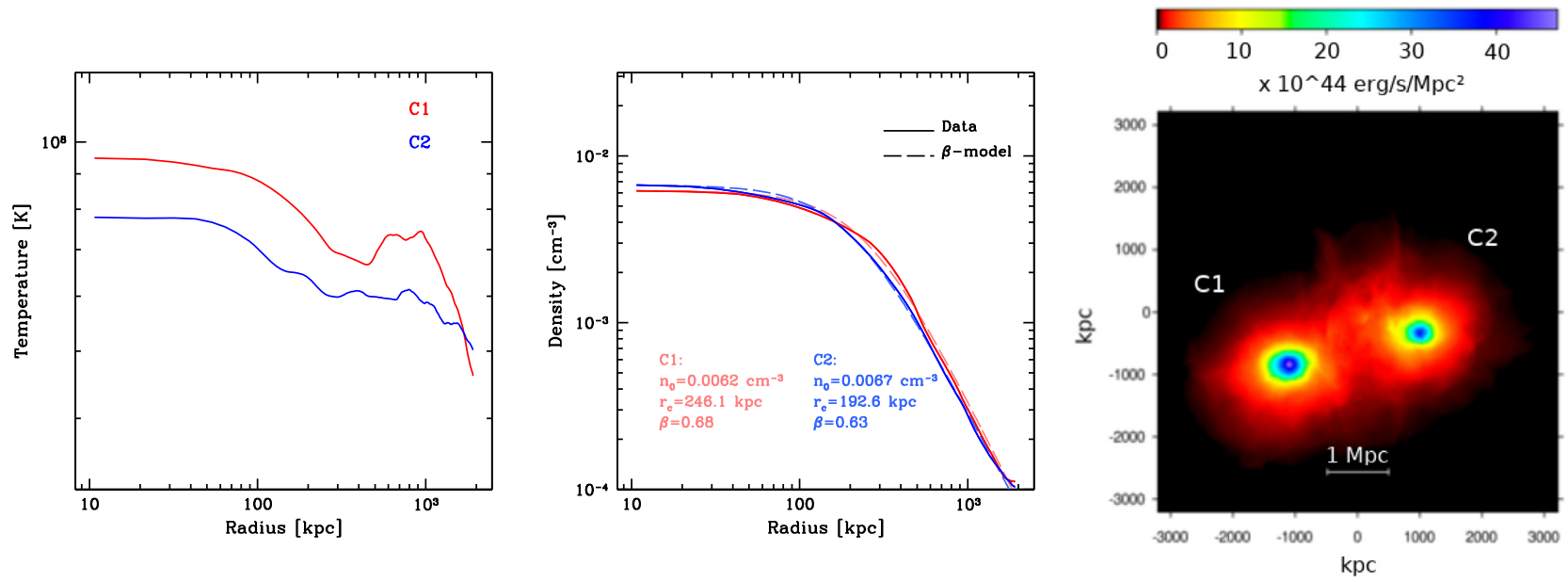

Figure 1. From left to right: temperature and density profiles for the $\mathrm{C} 1$ (red) and $\mathrm{C} 2$ (blue) clusters and X-ray surface brightness of the two clusters in the $0.1-2.4 \mathrm{keV}$ band. For the density profile, the best-fitting equations of a $\beta$ model are plotted as dashed lines, with parameters reported at the bottom of the plot. 

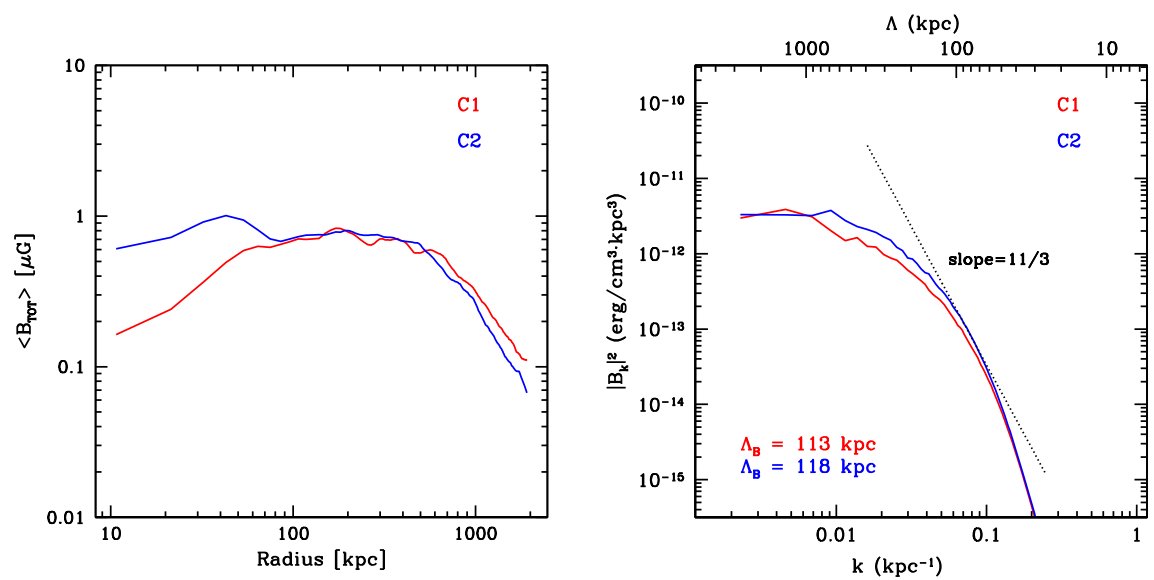

Figure 2. Left: average of the total magnetic field 3D profiles for the $\mathrm{C} 1$ (red) and $\mathrm{C} 2$ (blue) clusters. Right: magnetic field power spectra for the C1 (red) and $\mathrm{C} 2$ (blue) clusters. The autocorrelation length values are shown in the bottom left corner. A dotted black line shows a power spectrum with spectral index equal to $11 / 3$.
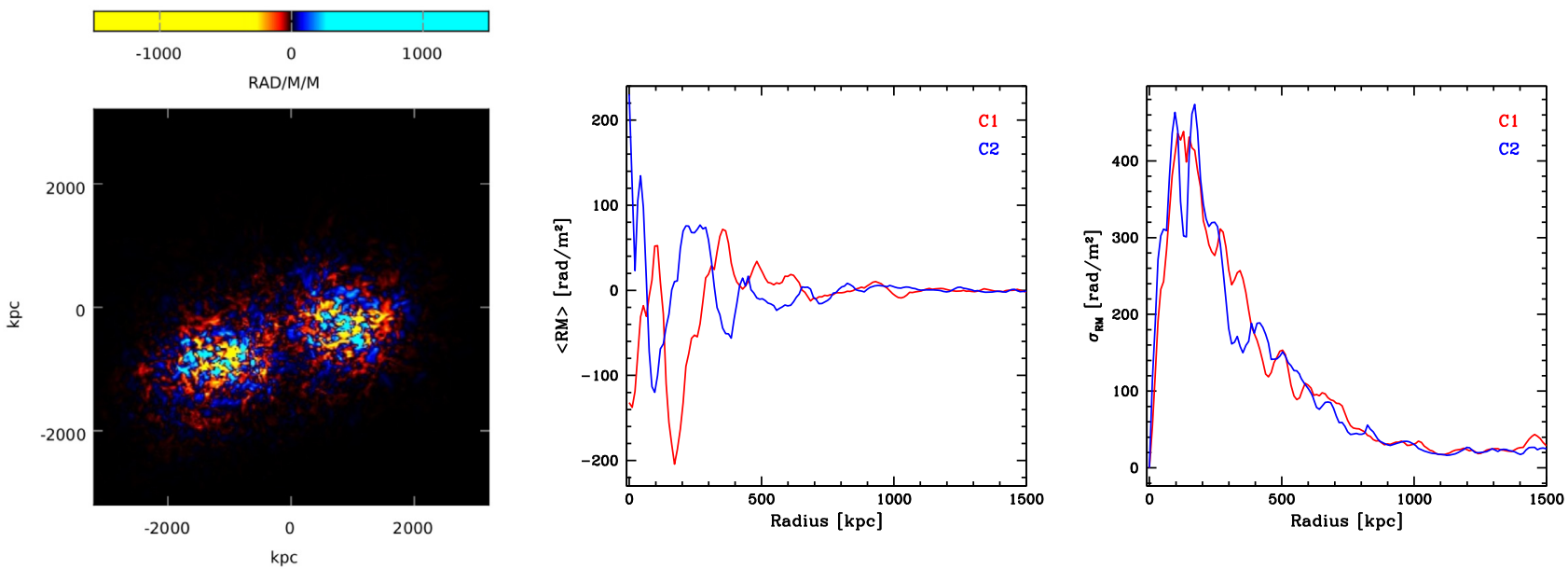

Figure 3. From left to right: intrinsic RM image computed from the MHD cubes, azimuthally averaged profiles in circular annuli of the mean RM and $\sigma_{\mathrm{RM}}$ as a function of distance from the cluster centres, in red for the $\mathrm{C} 1$ cluster and in blue for the $\mathrm{C} 2$ cluster.

and their values are reported in the bottom left corner of the plot. For the sake of comparison, a dotted black line traces a Kolmogorov-like power spectrum (slope equal to 11/3).

The cluster RM can be computed from equation (2) with the integral performed across the entire MHD box size. The resulting image is shown in Fig. 3 (left panel), together with the profile of the mean RM (middle panel) and its standard deviation $\sigma_{\mathrm{RM}}$ (right panel). This image suggests a turbulent magnetic field structure. From the profiles, we can observe large oscillations of the RM mean and high values of $\sigma$ near the centre, both decreasing as a function of distance. However, at the centre the profiles go to zero, due to the fact that the autocorrelation scale of the RM is larger than the size of the first annulus.

\subsection{FARADAY simulations}

The FARADAY software package (Murgia et al. 2004) has been specifically designed for intracluster magnetic field investigations. It can reproduce spectral cubes of the total and polarized intensity of simulated radio haloes (Murgia et al. 2004; Govoni et al. 2013), as well as the radio emission of a population of discrete radio sources (see Loi et al. 2019 for more details). Here, the discrete radio sources populating the field of view are simulated according to Loi et al. (2019). The radio haloes are simulated under two assumptions: (1) equipartition between magnetic field and relativistic electron energy density voxel by voxel, consistently with the work of Govoni et al. (2013), and (2) coupling between the relativistic particles responsible for the radio halo emission and the thermal particles. In this latter scenario, the energy density of the relativistic particles constitutes 0.3 per cent of the thermal energy density. This factor has been chosen in order to obtain a radio power at $1.4 \mathrm{GHz}$ similar to that of radio haloes in equipartition conditions and is consistent with the upper limit set from $\gamma$-ray observations (ratio between the relativistic and thermal particle energy density less than 10 per cent: see Brunetti, Zimmer \& Zandanel 2017).

To complete these radio simulations and make them suitable in the case of a pair of galaxy clusters, it is necessary to include a cluster population of radio sources. 


\subsubsection{Cluster discrete radio sources}

The cluster population is added to the simulation as follows:

(1) two families of sources are considered, AGNs and starforming galaxies (SFGs);

(2) values for $r_{200}$ and the cluster volume are set;

(3) it is assumed that the spatial distribution of the radio sources follows a Navarro-Frank-White profile (Lin \& Mohr 2007) and that the luminosity and density of the cluster sources are described by the radio luminosity functions of the same work;

(4) the Navarro-Frank-White distribution is normalized so as to yield the same number of sources per $\mathrm{Mpc}^{3}$ provided by the radio luminosity function;

(5) the total number of cluster sources $N$ is computed from the integration of the spatial distribution over the cubic box;

(6) the number of AGNs or SFGs is computed by multiplying the total number $N$ by the ratio between the radio luminosity function of one specific type (AGN or SFG) with respect to the total radio luminosity function;

(7) by using a Monte Carlo approach, the position $(x, y, z)$ and the luminosity of each source are extracted from the corresponding cumulative distribution functions.

The size, morphology and spectropolarimetric properties of the sources are assigned as described in Loi et al. (2019) (see that article for more details). It is worth recalling that radio galaxies moving across the ICM typically show a distorted morphology caused by the jets bending. Such tailed radio galaxies are classified as narrowangle tail, where the jets of a Fanaroff-Riley (FR) type I (Fanaroff \& Riley 1974) are evidently bent, and wide-angle tail, which is less bent than the previous one. When the jets of narrow-angle tail galaxies point toward the same direction, the radio source is called a head-tail galaxy. The simulations presented in this article show cluster radio galaxies with this peculiar morphology.

\subsubsection{Polarized emission}

The polarized emission of radio sources and the resulting images are obtained through the following steps for each line of sight:

(i) from the intrinsic polarized intensity $\left\|p_{v}\right\|$ and angle $\psi_{0}$ of a radio source at a given depth $l$, the $Q_{v}(l)$ and $U_{v}(l)$ Stokes parameters are computed taking into account the corresponding Faraday depth (see equation 1), according to

$$
\begin{aligned}
& Q_{v}(l)=\frac{\left\|p_{v}\right\|}{\sqrt{\tan ^{2} 2 \Psi+1}}, \\
& U_{v}(l)=\frac{\left\|p_{v}\right\| \tan 2 \Psi}{\sqrt{\tan ^{2} 2 \Psi+1}},
\end{aligned}
$$

at every $v$ of the simulated bandwidth;

(ii) the $Q_{v}(l)$ and $U_{v}(l)$ contributions are then summed up along the line of sight:

$$
\begin{aligned}
Q_{v} & =\int_{0}^{L} Q_{v}(l) \mathrm{d} l, \\
U_{v} & =\int_{0}^{L} U_{v}(l) \mathrm{d} l .
\end{aligned}
$$

By repeating this procedure for each line of sight, it is possible to obtain cubes of the $Q_{v}$ and $U_{v}$ Stokes parameters. The polarized intensity images are obtained by averaging the $Q$ and $U$ cubes over the whole frequency band and then computing the polarized intensity using equation (3). The images are then produced taking into account the depolarization effects mentioned in Section 1. It is worth noticing that they correspond to the result of RM synthesis considering the polarized signal at a Faraday depth equal to zero. Such images will be compared in Section 5 with the linearly polarized images retrieved after the application of RM synthesis.

\section{TOTAL INTENSITY RADIO IMAGES}

Starting from the MHD cubes and making use of the source modelling implemented in FARADAY, it is possible to reproduce the radio emission of the sources populating the field of view of the simulated cubes considering a given observational set-up. In the following section, we show simulated and synthetic total intensity images. The first are at the full resolution allowed by the simulation, $\sim 5$ arcsec pixel $^{-1}$, and noise-free, while the second are convolved with a beam resolution of 10 arcsec and a thermal noise of $0.1 \mu \mathrm{Jy}_{\text {beam }}{ }^{-1}$ is added.

\subsection{Simulated images}

Fig. 4 (top panel) shows the total intensity emission produced from a data cube spanning the frequency band $950-1760 \mathrm{MHz}$ with a spectral resolution of $1 \mathrm{MHz}$. These simulated images are at the maximum resolution offered by the MHD simulations, i.e. $\sim 10.7 \mathrm{kpc}$, which corresponds to $\sim 5 \operatorname{arcsec}$ at the cluster redshift $z$ $=0.115$, where the thermal noise is zero.

The dynamical range is very high, going from the sub$\mu \mathrm{Jy} \operatorname{arcsec}^{-2}$ brightness of the most distant sources up to the mJy $\operatorname{arcsec}^{-2}$ level of the cluster sources. The maximum value of the surface brightness is $\sim 0.5 \mathrm{mJy} \mathrm{arcsec}{ }^{-2}$, but it is not represented in the colour bar, which would otherwise be highly saturated. Two magenta circles of radius $1.5 \mathrm{Mpc}$ are centred on the cluster centres. The magenta boxes in the images identify the four different cluster radio sources shown in the bottom panels: from left to right, FRII, head-tail, cluster wide-angle tail and FRII radio galaxy.

Fig. 5 shows the total intensity image again, this time with the addition of the radio haloes of the two clusters. In Fig. 5(a), we assume the equipartition condition between the energy density of relativistic particles and the intracluster magnetic field. In Fig. 5(b), we assume that the energy density of relativistic particles is 0.003 times the thermal plasma energy density, a factor chosen to obtain radio haloes with the same luminosity at $1.4 \mathrm{GHz}$. In these images, the colour-bar limits were chosen to improve the contrast, but the actual maximum pixel values can reach $\sim 0.5 \mathrm{mJy} \operatorname{arcsec}^{-2}$. The surface brightness of radio haloes presents high values at the centre, between 0.3 and $0.7 \mu \mathrm{Jy} \operatorname{arcsec}^{-2}$, decreasing outwards. The filamentary morphology of the simulated radio haloes reflects the intracluster magnetic field structure, especially when equipartition is assumed. The radio haloes generate coupling between the thermal and non-thermal particle energy density to create a smoother morphology. This is because the emissivity of radio haloes in equipartition, assuming $\alpha=1$, is proportional to $B^{4}$, while it is proportional to $B^{2}$ for radio haloes coupled with thermal particles. This results in the emission of radio haloes under equipartition tracing the turbulent nature of the magnetic field more dramatically.

The properties of the simulated radio haloes are similar to those observed in terms of radio power at $1.4 \mathrm{GHz}$ and $\mathrm{X}$-ray luminosity. This is shown in the right panel of Fig. 5, where the two open circles and crosses correspond to the radio haloes in equipartition and coupled with the thermal plasma, respectively, in red for $\mathrm{C} 1$ and in blue for $\mathrm{C} 2$ cluster haloes, while the black points represents 

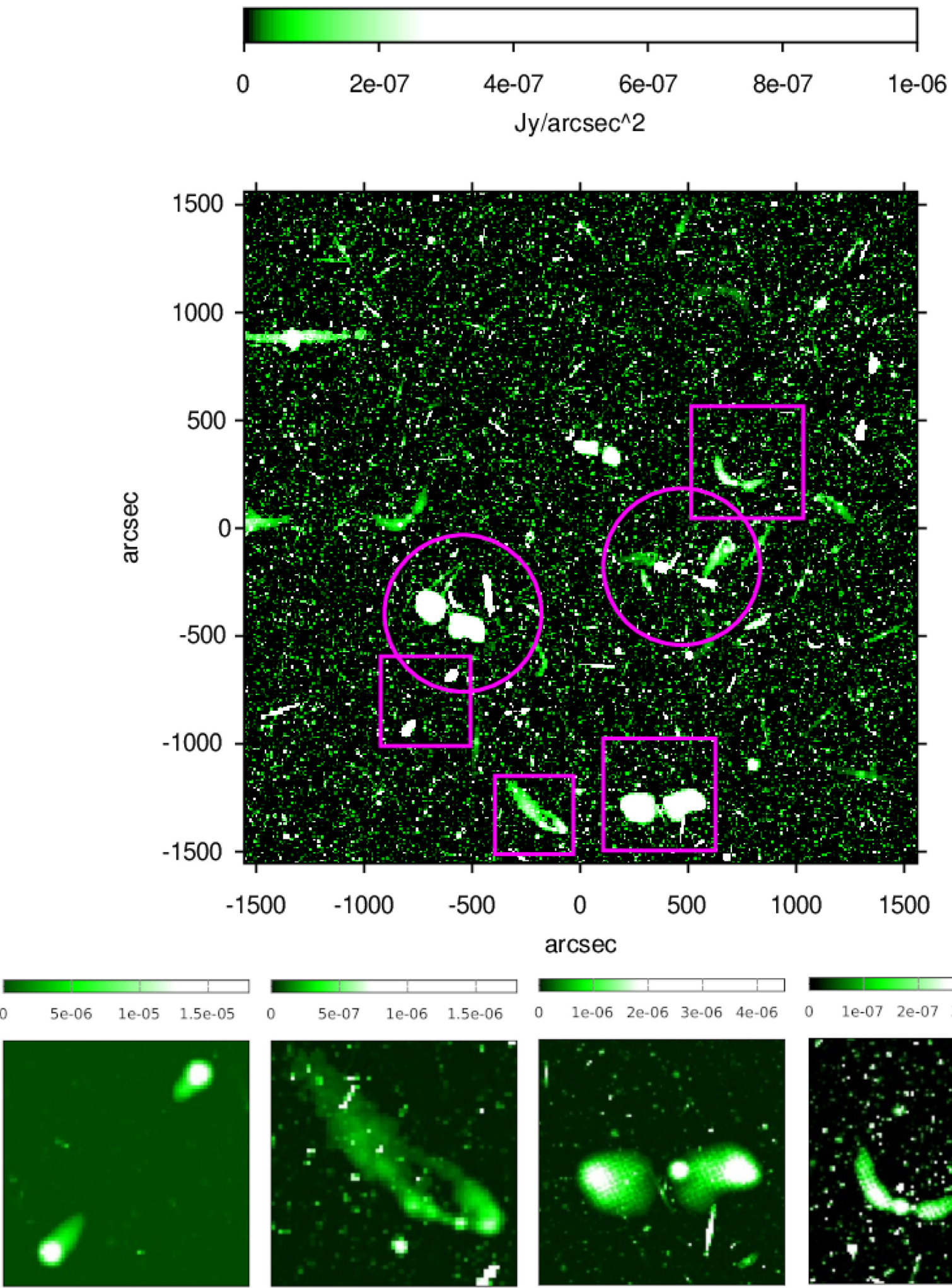

$0 \quad 1 \mathrm{e}-06 \quad 2 \mathrm{e}-06 \quad 3 \mathrm{e}-06 \quad 4 \mathrm{e}-06$

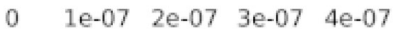

Figure 4. 1.4-GHz total intensity image of the two simulated galaxy clusters with a cell size of $\sim 5$ arcsec. The magenta circles are centred on the cluster centres and have a radius of $1.5 \mathrm{Mpc}$. The magenta boxes indicate four different cluster radio sources that are shown in the bottom zoomed panels (see the text for more details). The surface brightness in the images goes from 0 up to $\sim 0.5 \mathrm{mJy} \operatorname{arcsec}^{-2}$ and is obtained by spectral averaging all the frequency channels. 

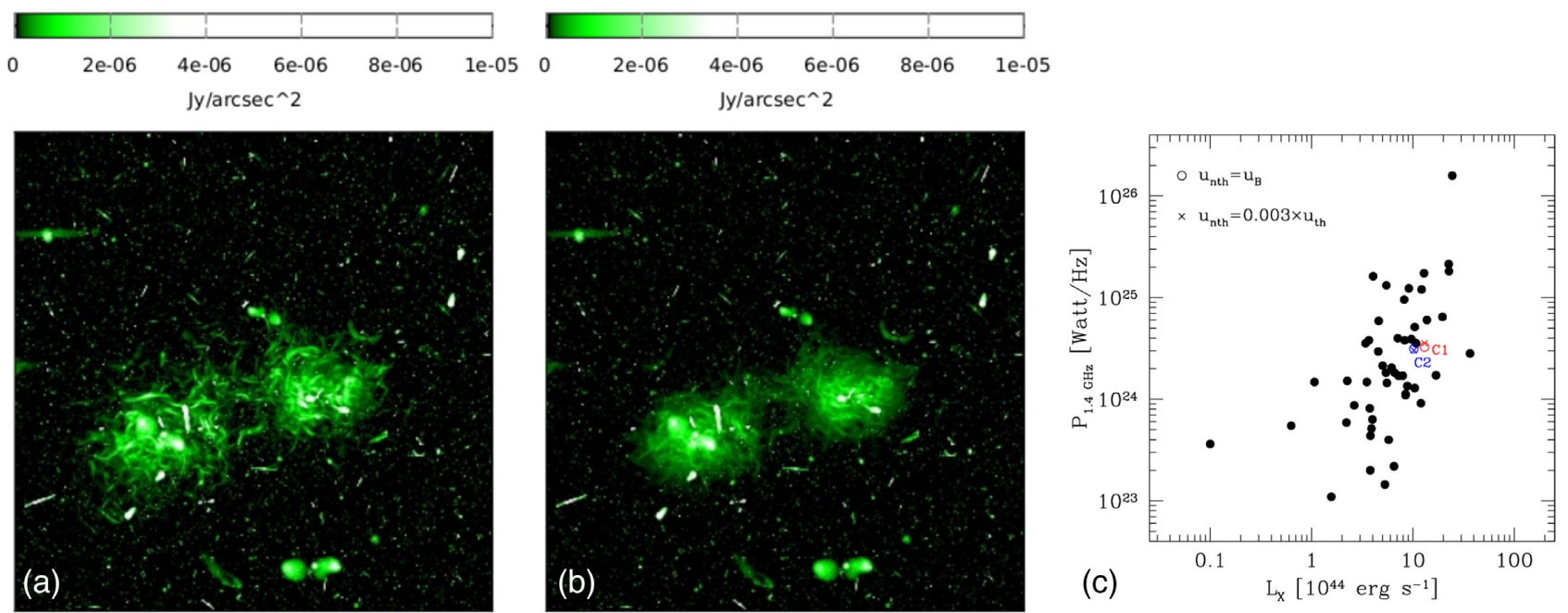

Figure 5. 1.4-GHz total intensity image of the two simulated galaxy clusters with a cell size of $\sim 5$ arcsec, where (a) radio haloes are simulated according to the equipartition condition and (b) the relativistic particle energy density is 0.3 per cent of the thermal plasma energy density. Panel (c) shows the 1.4-GHz power versus X-ray luminosity of several haloes reported in the literature (black) and those simulated here in red for the $\mathrm{C} 1$ cluster and in blue for the $\mathrm{C} 2$ one. Open circles and crosses refer to the two assumptions made to simulate the radio haloes. The surface brightness in the images goes from 0 up to $\sim 0.5 \mathrm{mJy}$ arcsec ${ }^{-2}$.

the results obtained for several observed radio haloes (see the compilation of Feretti et al. 2012; Govoni et al. 2012; van Weeren et al. 2012; Giovannini et al. 2013; Martinez Aviles et al. 2016; Shakouri, Johnston-Hollitt \& Pratt 2016; Parekh et al. 2017; Loi et al. 2017). It is worth noting that, even if the integrated values of the 1.4-GHz radio power are the same under both assumptions, the surface brightness of the radio halo simulated coupling between relativistic and thermal particles shows a rapid decrease going outwards, whereas under equipartition bright filaments are still visible at distances larger than $1.5 \mathrm{Mpc}$ from the cluster centres.

\subsection{Synthetic radio images}

Fig. 6 shows synthetic radio images of the two simulated clusters produced from a data cube spanning the frequency band 950$1760 \mathrm{MHz}$ with a channel resolution of $1 \mathrm{MHz}$. These synthetic cubes correspond to the simulated cubes of the previous subsection convolved with a Gaussian function having FWHM $=10$ arcsec, with the addition of thermal noise of $0.1 \mu \mathrm{Jy}$ beam $^{-1}$. This corresponds to the sensitivity level of $Q$ and $U$ Stokes images achievable with SKA1-MID with an integration time of $60 \mathrm{~h}$. It is worth specifying that, in total intensity, the confusion noise is significantly higher $\left(\sigma_{\mathrm{c}}=4 \mu \mathrm{Jy} \mathrm{beam}^{-1}\right.$ according to Loi et al. 2019) and is reached after a few minutes of observing time. In practice, the actual noise in these images is therefore dominated by the confusion noise. Fig. 6 shows the 1.4-GHz total intensity synthetic SKA1-MID images with (a) radio haloes produced assuming the equipartition condition and (b) a relativistic particle energy density equal to 0.3 percent of the thermal plasma energy density. Even in these images, the pixel range has been reduced, but the surface brightness can be as high as $0.16 \mathrm{mJy} \mathrm{beam}^{-1}$.

The radio haloes show a mean surface brightness computed in a circle of $1 \mathrm{Mpc}$ with a radius of $\sim 40 \mu \mathrm{Jy}_{\text {beam }}{ }^{-1}$. The filamentary structure of radio haloes in equipartition is still visible. On the other hand, the radio haloes simulated coupling between thermal and nonthermal particle energy densities present a smoother morphology. These results highlight how high-resolution and high-sensitivity observations performed with next-generation instruments such as
SKA1-MID could help us in understanding the nature of relativistic particles in cluster radio haloes, which is still an open and debated question in the literature.

\section{RM SYNTHESIS APPLICATION}

RM synthesis is one of the most used techniques to recover polarized signals and their associated Faraday depths in spectropolarimetric observations. Following the formalism adopted in Brentjens \& de Bruyn (2005), we will start by going over some basic concepts. We will follow by explaining the method chosen to interpret the output data.

\subsection{Basics and set-up}

Each line of sight where one or more polarized signals cross a magnetoionic medium is characterized by the so-called Faraday dispersion function $F(\phi)$, which represents the emitted polarization as a function of the Faraday depth $\phi$. With RM synthesis, it is possible to retrieve an approximate reconstruction of this function $\tilde{F}(\phi)$ that corresponds to the convolution of $F(\phi)$ and the RM transfer function $R(\phi)$ (RMTF), defined as

$R(\phi)=\frac{\int_{-\infty}^{+\infty} W\left(\lambda^{2}\right) \mathrm{e}^{-2 \mathrm{i} \phi \lambda^{2}} \mathrm{~d} \lambda^{2}}{\int_{-\infty}^{+\infty} W\left(\lambda^{2}\right) \mathrm{d} \lambda^{2}}$,

where the sampling function or weight function $W\left(\lambda^{2}\right)$ is non-zero at the measured $\lambda^{2}$ and zero elsewhere. Indeed, the approximate reconstruction of $F(\phi)$ is obtained by Fourier-transforming the observed polarized intensity as a function of $\lambda^{2}, \tilde{P}\left(\lambda^{2}\right)$ :

$\tilde{F}(\phi)=\frac{\int_{-\infty}^{+\infty} \tilde{P}\left(\lambda^{2}\right) \mathrm{e}^{-2 i \phi \lambda^{2}} \mathrm{~d} \lambda^{2}}{\int_{-\infty}^{+\infty} W\left(\lambda^{2}\right) \mathrm{d} \lambda^{2}}$.

All these functions are complex and $\tilde{P}\left(\lambda^{2}\right)=Q\left(\lambda^{2}\right)+\mathrm{i} U\left(\lambda^{2}\right)$. Equation (9) corresponds to a derotation of the $Q$ and $U$ Stokes parameters, assuming a given value of Faraday depth in a given interval and performed channel by channel, pixel by pixel. The resulting $Q$ and $U$ Stokes parameter cubes as a function of the 

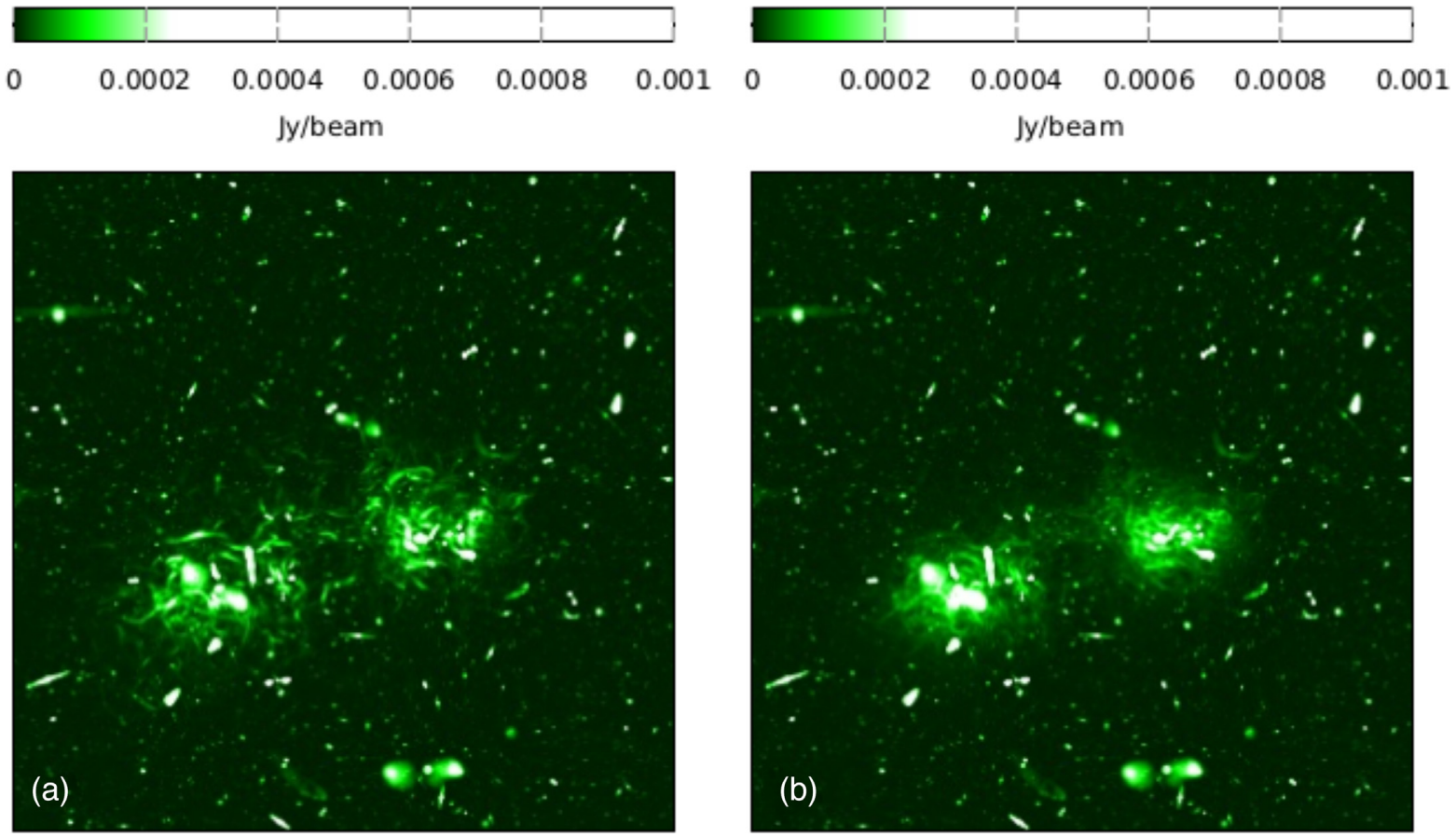

Figure 6. 1.4-GHz total intensity synthetic SKA1-MID images obtained by degrading the resolution of the simulated galaxy clusters (see Fig. 4) to 10 arcsec and adding $0.1 \mu \mathrm{Jy}_{\text {beam }}{ }^{-1}$ noise. The cluster radio haloes are produced (a) assuming the equipartition condition and (b) assuming a relativistic particle energy density equal to 0.3 per cent of the thermal plasma energy density. The surface brightness in the images goes from 0 up to $\sim 0.16 \mathrm{mJy}^{\text {beam }}{ }^{-1}$.

Faraday depth can be used to build a polarized intensity cube as shown in equation (3) by taking the modulus of the Faraday dispersion function.

The accuracy of the RM-synthesis technique is determined by the $\lambda^{2}$ coverage $\Delta \lambda^{2}$, the minimum wavelength squared $\lambda_{\min }^{2}$ and the channel width $\delta \lambda^{2}$. In particular, their values define three important parameters: (a) the resolution in Faraday space (which corresponds to the FWHM of the RMTF), (b) the largest scale in Faraday depth and (c) the maximum observable Faraday depth to which the technique is sensitive. For a bandwidth between 950 and $1760 \mathrm{MHz}$ with a frequency channel of $1 \mathrm{MHz}$, the former parameters become

$$
\begin{aligned}
\delta \phi & \approx \frac{2 \sqrt{3}}{\Delta \lambda^{2}}=49 \mathrm{rad} \mathrm{m}^{-2}, \\
\max \text {-scale } & \approx \frac{\pi}{\lambda_{\text {min }}^{2}}=108 \mathrm{rad} \mathrm{m}^{-2}, \\
\left|\phi_{\max }\right| & \approx \frac{\sqrt{3}}{\delta \lambda^{2}} \approx 24000 \mathrm{rad} \mathrm{m}^{-2},
\end{aligned}
$$

where $\delta \lambda^{2}$ is computed at the centre of the bandwidth. This observational set-up is a compromise between minimizing the RMTF FWHM (and therefore Faraday-space resolution), which improves at lower frequencies, and the need for sensitivity to large-scale structures in Faraday space, which improves at high frequencies. Indeed, with a frequency band between 350 and $1050 \mathrm{MHz}$, the resolution and maximum scale are, respectively, 5 and $38 \mathrm{rad} \mathrm{m}^{-2}$. For example, a radio halo filament having a width of $50 \mathrm{kpc}$ located in the plane of the sky with a thermal density of $0.006 \mathrm{~cm}^{-3}$ and a magnetic field of $0.5 \mu \mathrm{G}$ would produce a Faraday thick region of $\sim 120 \mathrm{rad} \mathrm{m}^{-2}$. This quantity roughly corresponds to the maximum scale measurable in Faraday depth between 750 and
$1760 \mathrm{MHz}$, while it is three times the maximum scale between 350 and $1050 \mathrm{MHz}$.

The Faraday space in this work is sampled with a cell size of $10 \mathrm{rad} \mathrm{m}^{-2}$ between -2000 and $2000 \mathrm{rad} \mathrm{m}^{-2}$, in order to have $\sim 5$ pixels in the RMTF FWHM. The weight function is assumed to be equal to 1 for each observed $\lambda^{2}$ and zero elsewhere.

\subsection{Interpretation of the products}

As mentioned in the previous sections, the total intensity images of this work, unlike the polarized intensity ones, are confusionlimited. Therefore, there will be polarized sources above the noise level in polarization which are not distinguishable from the noise in the total intensity images. The usual approach is to perform a cut on the RM-synthesis output images by creating a $3 \sigma_{I}$ mask with the total intensity map, but this will significantly limit the information potentially available in polarization. Another issue related to the interpretation of the RM-synthesis results is the question of obtaining the polarized intensity from the cubes. Since there could be, especially in the case of radio haloes, much polarized emission along the line of sight, the result is that the Faraday dispersion function is likely to feature more than one peak. The resulting polarization is then the sum of the contribution along the spectrum. However, the polarized intensity cube contains a positive bias, due to the fact that it is the square root of the sum of two squares $\left(\|P\|=\sqrt{Q^{2}+U^{2}}\right)$, which produces a mean $\|P\|>0$ even in the absence of source signals because of the presence of thermal noise. The plateau in the polarization image obtained by summing up all the polarization as a function of the Faraday depth can be significantly high, as it propagates the positive bias a number of times equal to the Faraday depth channels, resulting in a very low signal-to-noise $(\mathrm{S} / \mathrm{N})$ ratio. 
In order to avoid the emergence of a positive bias and the loss of polarized sources above the $3 \sigma_{I}$ level in polarization, in this work the polarized intensity cube is treated as follows.

(i) First, a mean is evaluated from the first channel of the polarized intensity cube as a function of the Faraday depth where $\phi=-2000 \mathrm{rad} \mathrm{m}^{-2}$ and it is unrealistic to have a real cluster RM. Indeed, to obtain such values, for a thermal density of $\sim 10^{-3} \mathrm{~cm}^{-3}$ the magnetic field should be of the order of a few $\mathrm{mG}$, which is three orders of magnitudes larger than the typical intracluster magnetic field strength. Therefore, it is possible to evaluate a mean over the full image in polarization at $\phi=-2000 \mathrm{rad} \mathrm{m}^{-2}$ and subtract it from each pixel of the polarized intensity cube as a function of the Faraday depth. The result is that the spectra along $\phi$ will scatter around zero and the sum of the polarization contributions along $\phi$ will not be affected by positive bias.

(ii) Then, the polarized image is created by summing up all the contributions along $\phi$ of the polarized intensity cube corrected for the bias.

(iii) The value corresponding to the maximum peak in polarized intensity is considered to be the measured RM. This is clearly an arbitrary choice and there is no guarantee that it is the correct one for a given line of sight. Indeed, the cluster RM could be the Faraday depth associated with lower polarized emission. A choice of some kind must be made, however: this is one of the most important issues in the interpretation of this technique and it will be the subject of future work. The resulting RM images are multiplied by a factor ( 1 $+z)^{2}$, with $z$ the cluster redshift, to correct for the Doppler effect on the observed frequencies.

(iv) As a final step, it is necessary to filter the polarization and $\mathrm{RM}$ images, selecting the pixels where the information is related to a real signal. To do this in the simulated images, we create a mask where the polarized intensity is different from zero and we use this mask to blank the RM images. In the synthetic images, the procedure is not so straightforward, because of the presence of noise. We thus use an iterative process to evaluate the root-meansquare (rms) value in a region of the polarization image where no obvious sources are present, clipping the pixels above three times the rms. The mask is then created by selecting all pixels with signal four times above rms. The factor 4 has been chosen as a compromise between the need to discard noise signals and the need to preserve as many pixels as possible.

\section{RM SYNTHESIS RESULTS}

In this section, we show the results of the procedure described previously, focusing on linearly polarized emission and the RM images obtained from simulated and synthetic data.

\subsection{Polarization results}

Figs 7 and 8 show the result of the RM-synthesis technique on simulated and synthetic data. The first rows show the observed linearly polarized intensity computed assuming a RM equal to zero, while the second rows show the linearly polarized intensity retrieved after the application of RM synthesis. From left to right, the panels refer to clusters without radio haloes, with radio haloes under the equipartition condition and assuming coupling between non-thermal and thermal particle energy density.

Simulated images of clusters without radio haloes show the presence of a large number of background sources that are below the noise level in the synthetic ones. This is mainly due to beam depolarization, which reduces the polarized signal of the faint background sources. Along with the presence of thermal noise, beam depolarization prevents the recovery of background signals in the synthetic images. Indeed, the radio sources near to the cluster centres in these images are cluster radio sources. We discuss the consequence of a very low background contribution in Section 6.

As for the total intensity emission, the linearly polarized emission suggests quite different morphology of the two kinds of simulated radio haloes: radio haloes in equipartition (Figs 7 and $8 \mathrm{~b}$ and e) shine in polarization even at large distances from the cluster centres, showing a discontinuous arrangement of bright and faint filaments, while radio haloes with coupling between thermal and non-thermal particles (Figs 7 and $8 \mathrm{c}$ and f) show a clear and regular decrease of the surface brightness from the centre to the outskirts and the filamentary morphology is less pronounced. As expected, the polarized intensity from RM synthesis is larger than that computed assuming $\mathrm{RM}=0$, in the case of both simulated and synthetic clusters, suggesting that bandwidth depolarization is a significant effect here. This is more evident in the central region of the clusters, where higher values of RM are responsible of the strong depolarization of $\sim 20-30$ per cent toward the centre for simulated images and $\sim 40-60$ per cent for synthetic ones. However, comparing between simulated and synthetic results, we can see that some polarized emission in synthetic data, especially that of the radio haloes on the outskirts, is lost, due to the fact that this emission does not emerge from the noise in the RM-synthesis cubes. Finally, it is important to notice how the presence of a large-scale diffuse source, i.e. the radio halo, is crucial in sampling the RM over a large area of the cluster: this will be possible with data acquired with SKA1-MID and analysed with the RM-synthesis technique.

\subsection{RM images}

Fig. 9 shows the results concerning the RM for simulated and synthetic data, respectively. The first rows refer to the intrinsic RM (computed from the MHD cubes), the second rows to the $\mathrm{RM}$ retrieved after the application of RM synthesis. From left to right, images show the RM of clusters without radio haloes, clusters with radio haloes in equipartition and simulated coupling between thermal and non-thermal particles. Similarly, the results for synthetic data are reported in Fig. 10.

At the cluster centres, the RM-synthesis RM values are lower in absolute value, in general, than the intrinsic ones. The central region of clusters is sampled in a better way in the case of simulated data with respect to the synthetic data, especially for clusters without radio haloes. This is caused by the presence of numerous background sources in the simulated images, which unfortunately are below the noise level in the synthetic ones. No substantial differences appear between the two kind sof simulated radio haloes, except for a slightly larger area sampled by the synthetic radio haloes in equipartition, due to the larger extension of the corresponding polarized emission.

\section{RM ANALYSIS}

The aim of this work is to compare the results of RM synthesis applied to SKA1-MID synthetic data with the input information and to analyse the discrepancies between the two. The determination of cluster magnetic fields here is based on RM properties. Indeed, the radial profile of $\sigma_{\mathrm{RM}}$ and the RM structure function, together with the fractional polarization, are the observables used to constrain the 

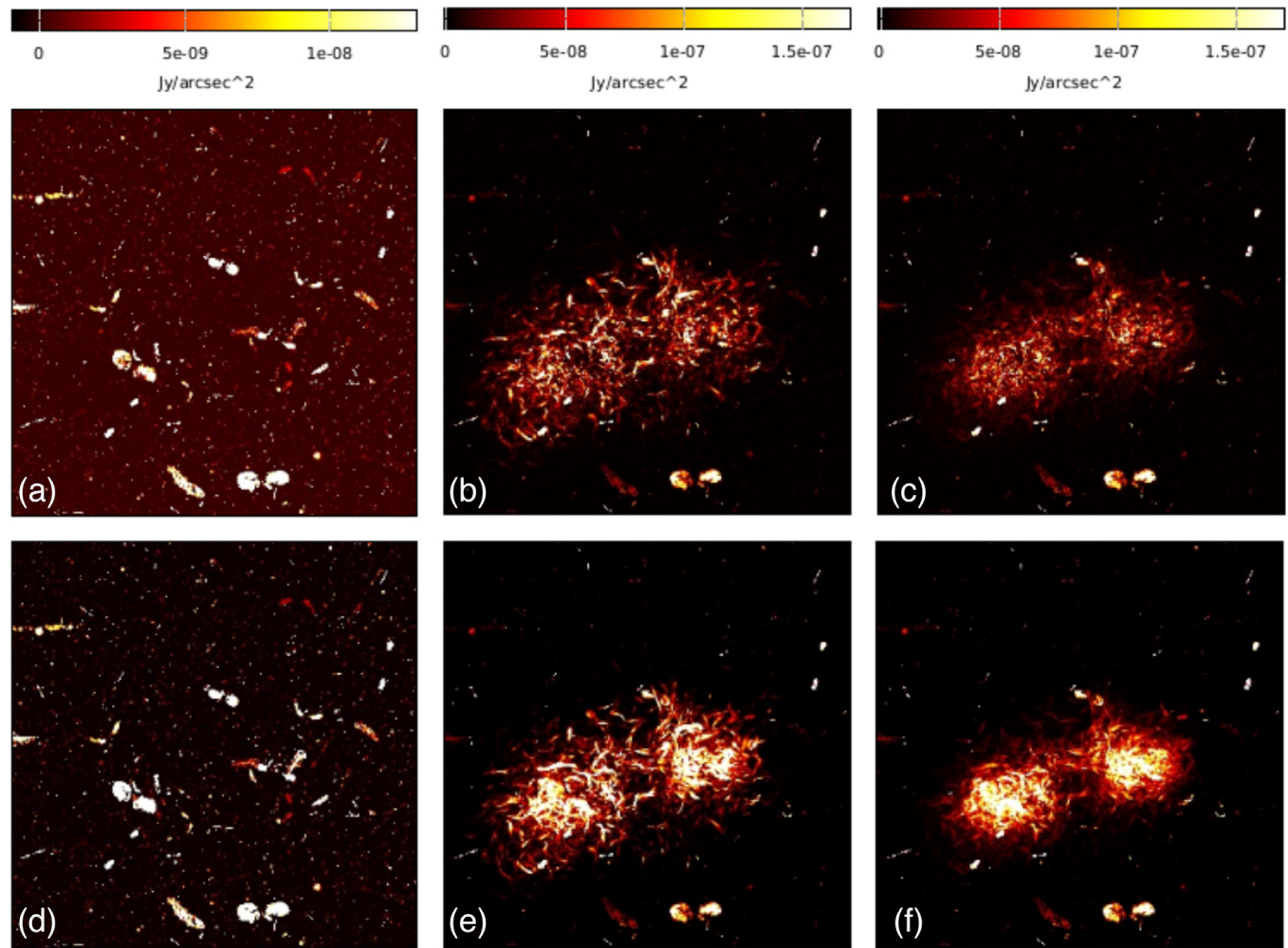

Figure 7. 1.4-GHz linearly polarized simulated images. The first row shows the linearly polarized intensity computed assuming RM $=0$, the second row the linearly polarized intensity reproduced after the application of RM synthesis. From left to right: clusters without radio haloes, with radio haloes under the equipartition condition and simulated coupling between thermal and non-thermal particles.
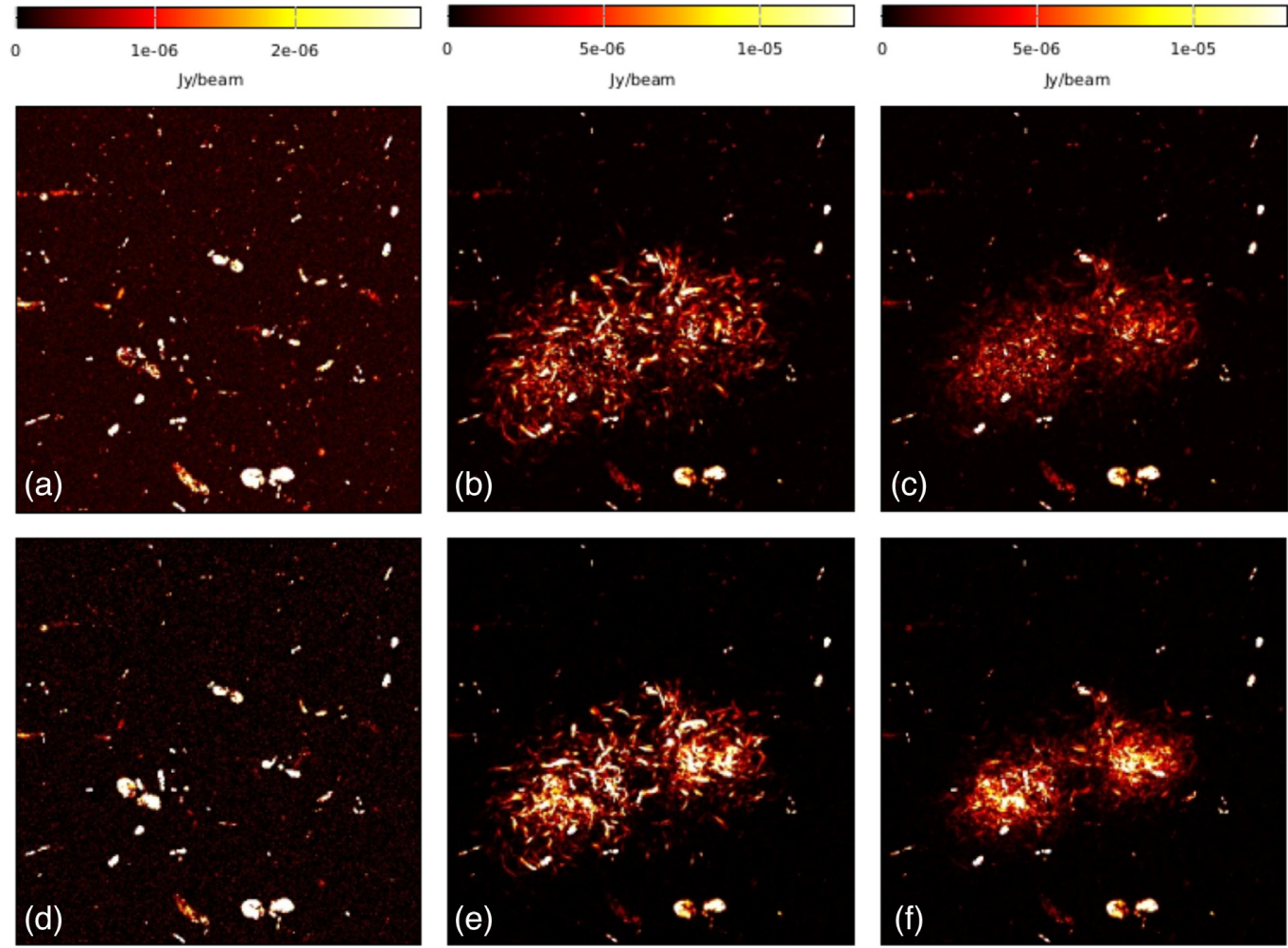

Figure 8. 1.4-GHz linearly polarized synthetic images. See caption of Fig. 7 for more details. 

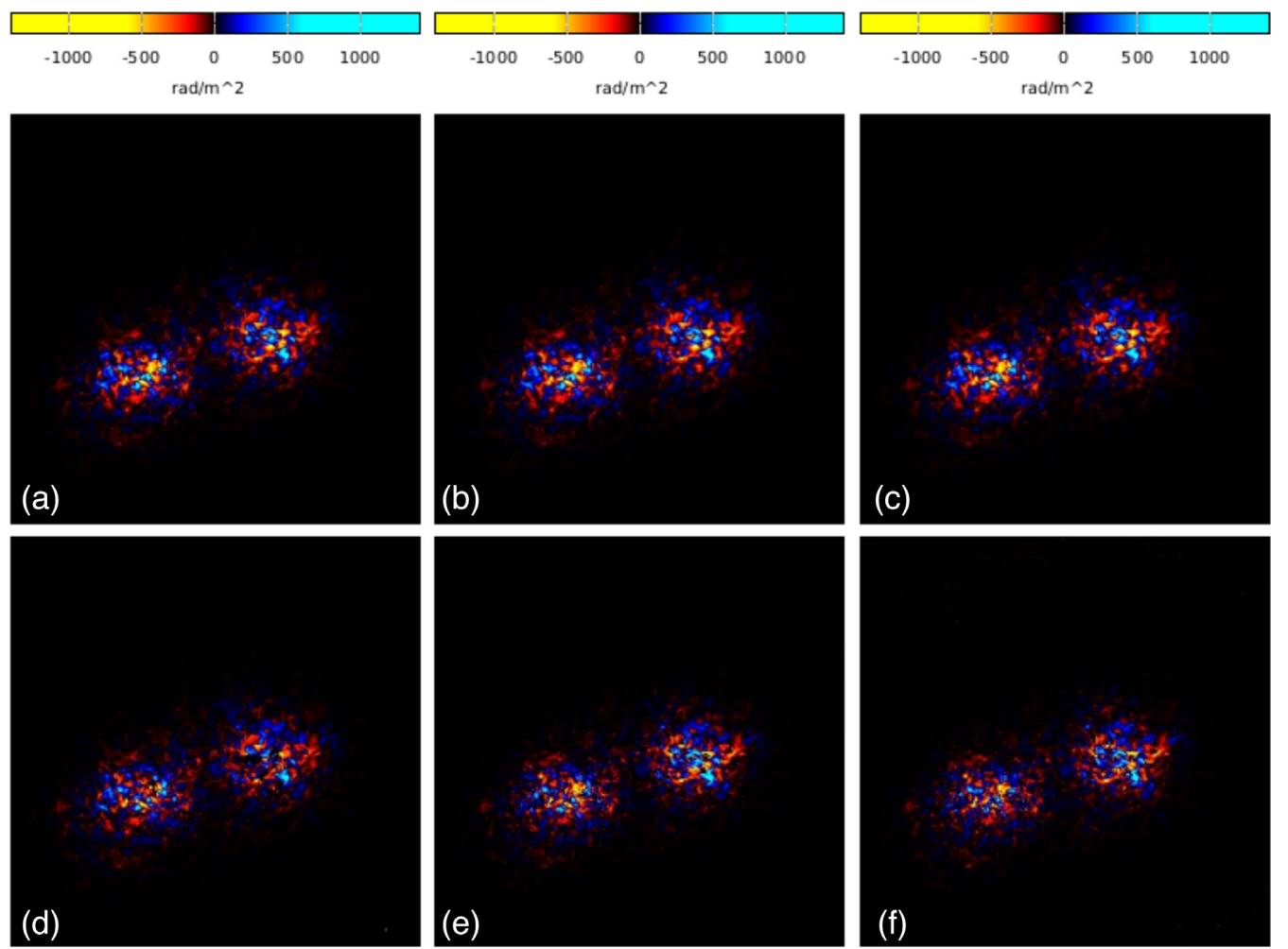

Figure 9. RM simulated images. The first and second rows refer respectively to the intrinsic RM (computed from the MHD cubes) and the RM retrieved after the application of RM synthesis. From left to right, images refer to clusters without radio haloes, radio haloes in equipartition and simulated coupling between thermal and non-thermal particles.
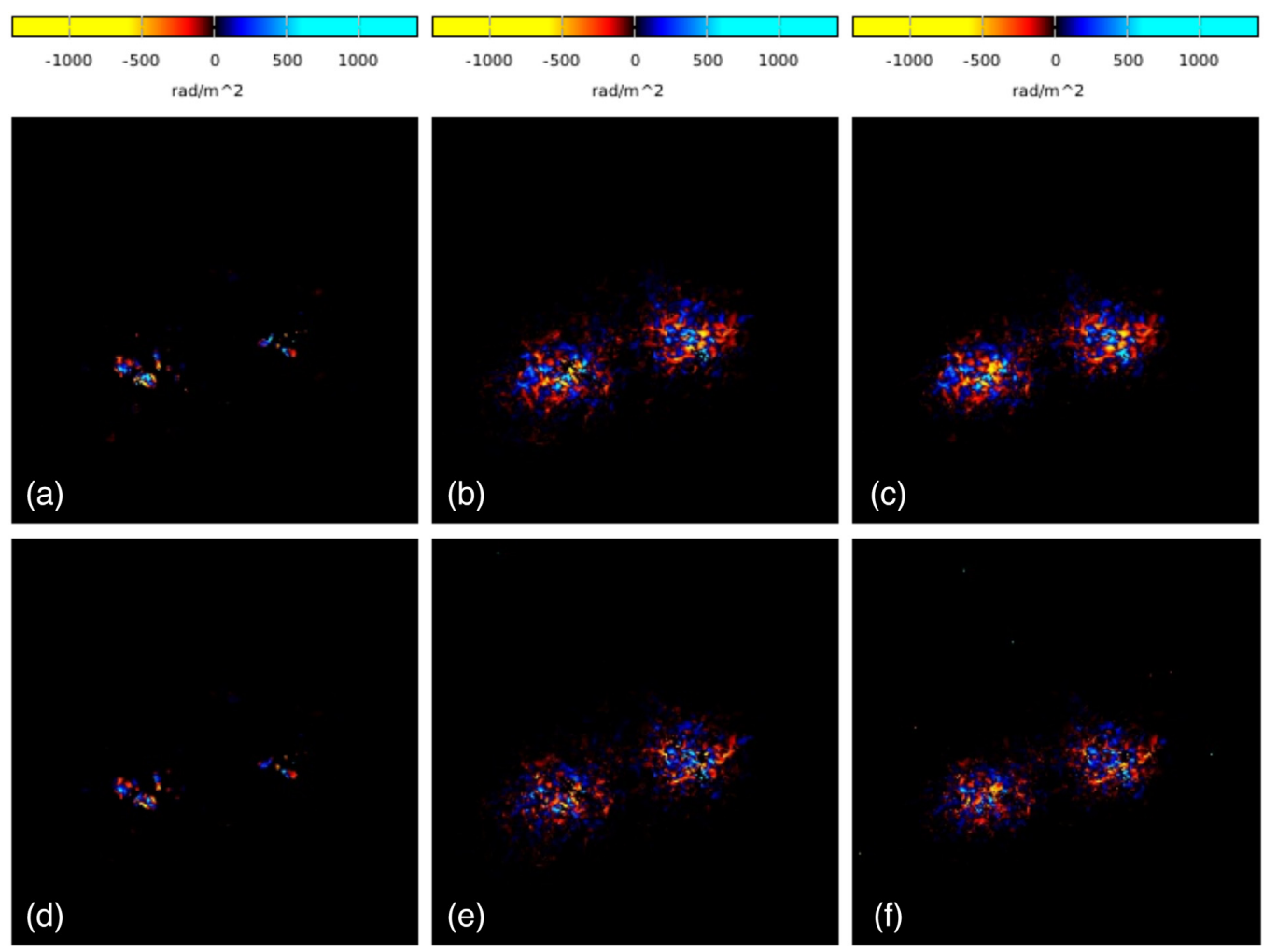

Figure 10. RM synthetic images. See caption of Fig. 9 for more details. 

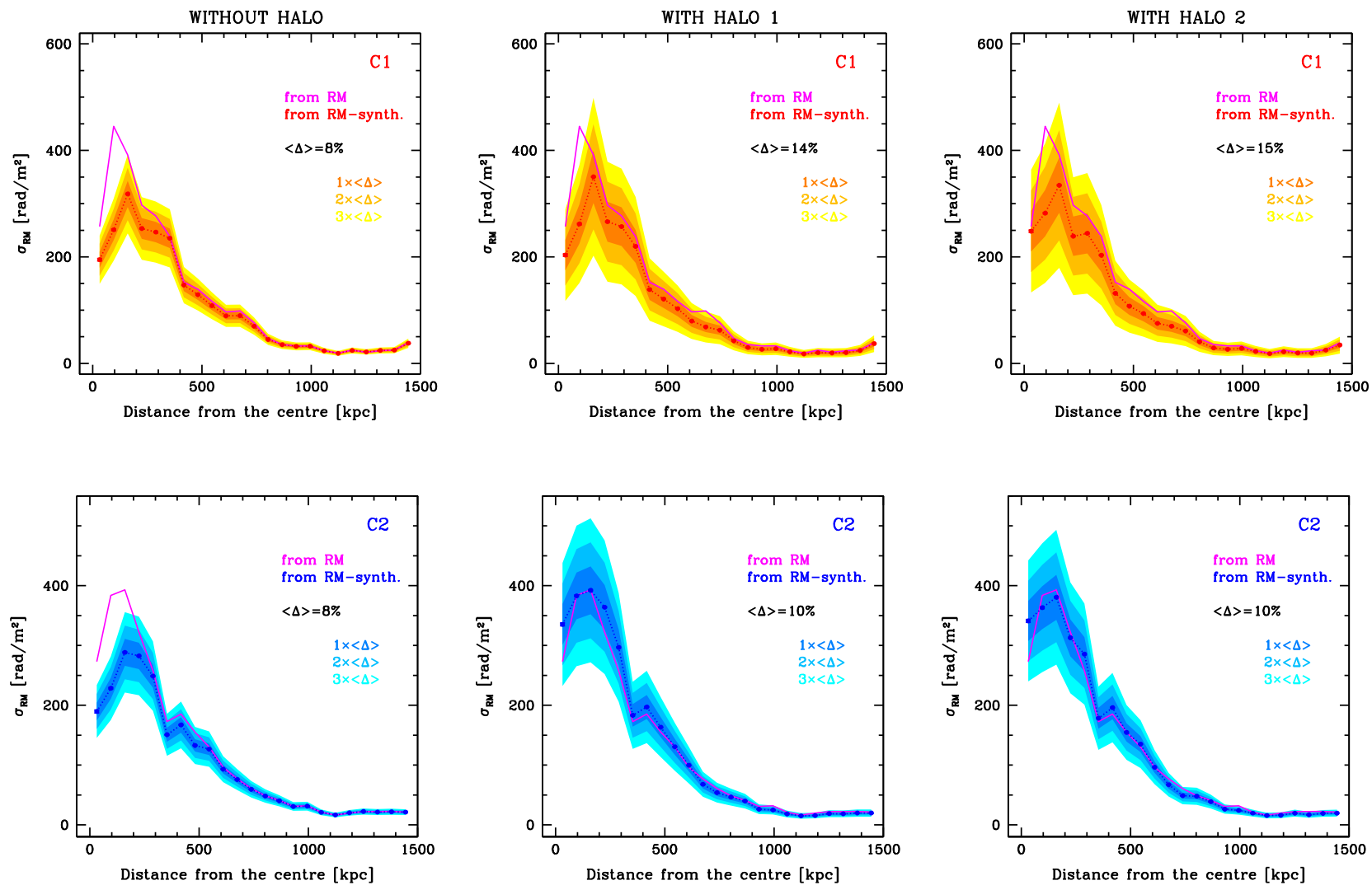

Figure 11. Simulated $\sigma_{\mathrm{RM}}$ profiles computed in annuli of 6 pixels width corresponding to a spatial width of $\sim 64 \mathrm{kpc}$. Top and bottom panels refer to the $\mathrm{C} 1$ and $\mathrm{C} 2$ simulated clusters. From left to right, results are shown for clusters without radio haloes, with radio haloes in equipartition and simulated by coupling non-thermal and thermal particles. In each panel, a solid magenta line represents the profile computed from the intrinsic RM images (Fig. 3) and points and dotted red lines refer to the profile computed from the output Faraday depth (bottom images of Fig. 9). $\langle\Delta\rangle$ indicates the average difference in per cent between the results of RM synthesis and intrinsic RM. Shadow regions are traced at 1,2 and $3 \times\langle\Delta\rangle$.

magnetic field power spectrum (e.g. Murgia et al. 2004; Govoni et al. 2006; Guidetti et al. 2008, 2010; Laing et al. 2008; Bonafede et al. 2010; Vacca et al. 2010, 2012; Govoni et al. 2017). In what follows, two different diagnostic quantities are analysed.

\subsection{The $\sigma_{\mathrm{RM}}$ profile}

The $\sigma_{\mathrm{RM}}$ profiles are computed from the Faraday depth images (bottom images of Figs 9 and 10) in annuli of 6 pixels width, corresponding to a spatial width of $\sim 64 \mathrm{kpc}(\sim 30 \operatorname{arcsec})$. The uncertainties associated with measurements of the output Faraday depth images are calculated as follows (Sotomayor-Beltran et al. 2013):

$\Delta \phi(l)=\frac{1}{S / N} \frac{\sqrt{3}}{\lambda_{2}^{2}-\lambda_{1}^{2}}$,

where $\lambda_{2}$ and $\lambda_{1}$ are the maximum and minimum wavelengths of the bandwidth, respectively, and $\mathrm{S} / \mathrm{N}$ the signal-to-noise ratio (here, equal to 1). Therefore, the uncertainty in $\sigma_{\mathrm{RM}}$ is

$\Delta \sigma_{\mathrm{RM}}=\sqrt{\sum_{i}\left(\frac{\partial \sigma_{R M}}{\partial \phi_{i}(l)} \Delta \phi_{i}(l)\right)^{2}}=\frac{\Delta \phi(l)}{\sqrt{N}}$,

where $N$ is the number of pixels considered to compute the corresponding $\sigma_{\mathrm{RM}}$.

Figs 11 and 12 show the $\sigma_{\text {RM }}$ profile from simulated and synthetic images, respectively. Top and bottom panels refer to the $\mathrm{C} 1$ and $\mathrm{C} 2$ simulated clusters. They are, from left to right, clusters without radio haloes, with radio haloes in equipartition and with simulated coupling between non-thermal and thermal particles. In each panel, a solid magenta line represents the profile computed from the intrinsic RM images (Fig. 3) and points and dotted lines refer to the profile computed from the output Faraday depth (bottom images of Fig. 9).

Simulated data show some deviations with respect to the intrinsic $\sigma_{\mathrm{RM}}$ values near to the cluster centre. These central decrements are due to a different distribution of intrinsic RM values with respect to the RM-synthesis RMs, as shown in Fig. 13 for the simulated $\mathrm{C} 1$ cluster without radio halo. In this plot, the RM values are compared point to point within a distance of $300 \mathrm{kpc}$ from the cluster centre. The RM distributions are projected on to the axis, along with the mean and rms of each distribution. The RM-synthesis histogram is more peaked near to zero than the intrinsic one, which is consistent with having lower values of $\sigma_{\mathrm{RM}}$ at distances less than $300 \mathrm{kpc}$. Clusters with radio haloes show similar histograms, including the $\mathrm{C} 2$ cluster hosting a radio halo, for which the intrinsic and simulated $\sigma_{\mathrm{RM}}$ are alike. For all three cases under study, the two histograms start to be more consistent at distances larger than $300 \mathrm{kpc}$, where intrinsic RM values are lower. The source position with respect to the cluster centre is probably responsible for the observed discrepancies. Grey crosses in Fig. 13 indicate dots that refer to background sources, which cover $\sim 30$ per cent of the cluster area within a radius equal to $300 \mathrm{kpc}$. In this plot, the crosses overlap each other over the RM value range, creating a continuous grey 

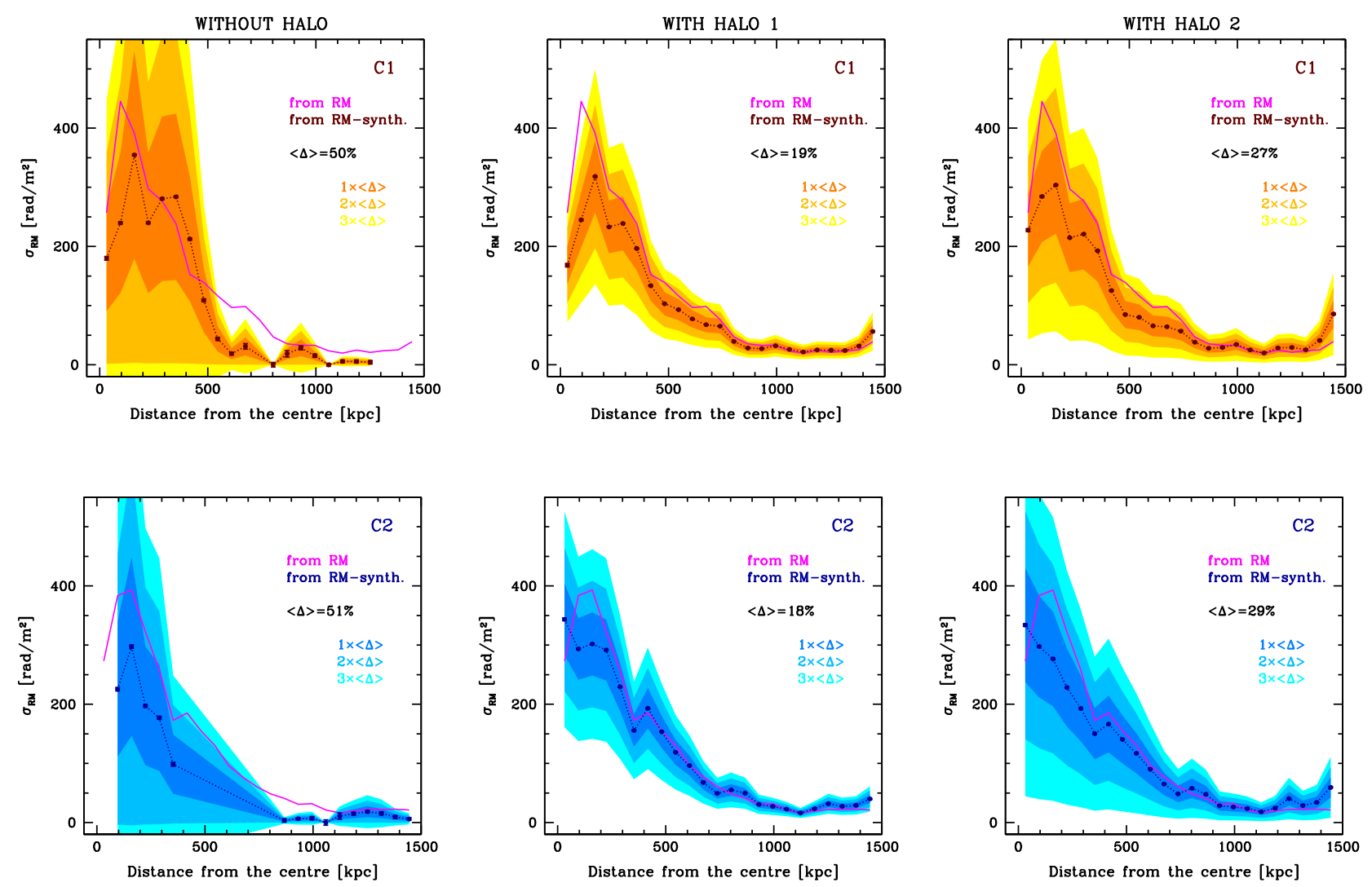

Figure 12. Synthetic $\sigma_{\mathrm{RM}}$ profiles computed in annuli of 6 pixels width corresponding to a spatial width of $\sim 64 \mathrm{kpc}$. See caption of Fig. 11 for more details.

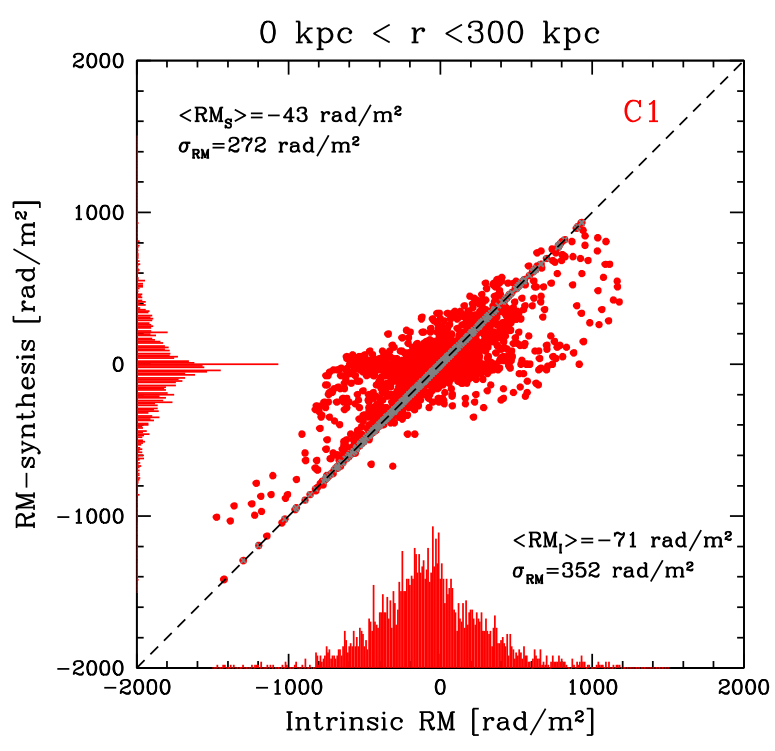

Figure 13. Point-to-point comparison between 0 and $300 \mathrm{kpc}$ from the cluster centre of intrinsic RM values ( $x$-axis) and those from RM synthesis ( $y$-axis) for the simulated $\mathrm{C} 1$ cluster without radio halo. Distribution histograms, along with the mean and rms, are also given. Grey crosses indicate the contribution of background sources.

region along the linear correlation drawn as a dashed black line. The perfect match between the intrinsic and RM-synthesis RM values supports the claim that the discrepancies are due only to the different paths crossed by linearly polarized signals along the line of sight.
Going from simulated to synthetic data, the $\sigma_{\mathrm{RM}}$ profiles change dramatically for clusters without radio haloes, compared with clusters with radio haloes. In simulated data, the entire cluster area is covered by background and cluster radio sources and, as shown in Fig. 13, the presence of background sources guarantees the recovery of the cluster RM. When thermal noise is added and the images are convolved at 10 arcsec, the background sources are too faint to survive in polarized images, mainly because of the beam depolarization. Therefore, the measured RM is associated mostly with cluster sources, as already mentioned in Section 5.1. Indeed, within a distance of $300 \mathrm{kpc}$ from the $\mathrm{C} 1$ cluster centre, there are no RMs associated with background sources, and they cover $\sim 7$ per cent of the cluster area up to a radius of $1500 \mathrm{kpc}$. Moreover, in this condition the background sources do not report the correct RM values, as shown in Fig. 14. The undersampling of the cluster area, mainly due to the low $\mathrm{S} / \mathrm{N}$ for background sources, and the source position can justify the irregular behaviour of the synthetic $\sigma_{\mathrm{RM}}$ profiles of clusters without radio haloes, where it is possible to observe both higher and lower values of $\sigma_{\mathrm{RM}}$ with respect to the intrinsic one.

Even clusters hosting radio haloes show a synthetic profile different from the simulated one, more evident in the case of the $\mathrm{C} 2$ rather than the $\mathrm{C} 1$ cluster, and, in particular, $\sigma_{\mathrm{RM}}$ presents lower values near to the centre. Here, the sampling problem is not observed and so we believe that this effect is due to both the presence of thermal noise, which as observed in the case of background sources can shift the peak in the Faraday dispersion function, and also the convolution of the $Q$ and $U$ data cubes with a Gaussian function having FWHM $=10$ arcsec, which decreases the polarized intensity and therefore increases the shifts produced by thermal noise. These 


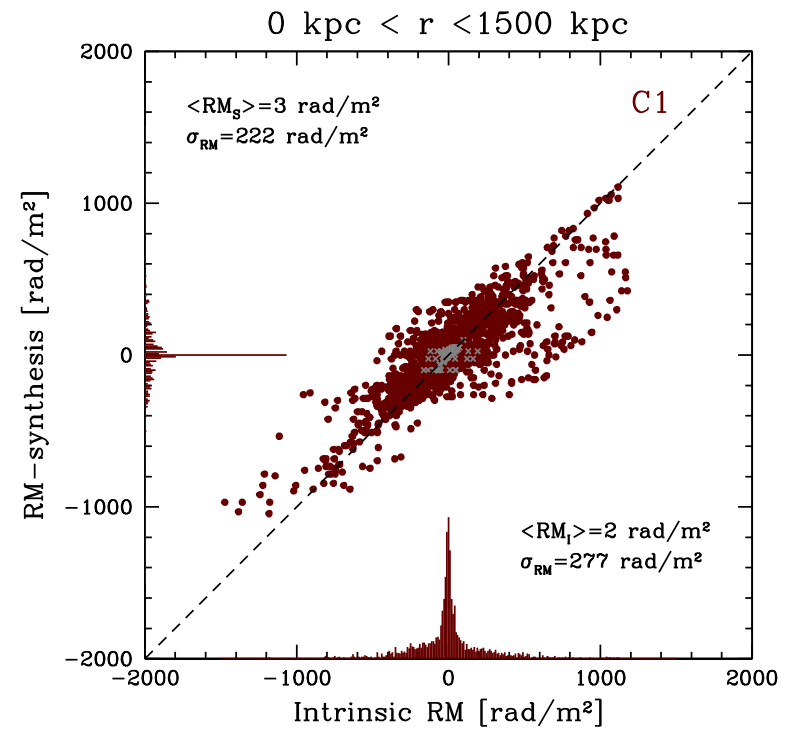

Figure 14. Point-to-point comparison between 0 and $1500 \mathrm{kpc}$ from the cluster centre of the intrinsic RM values ( $x$-axis) and those from RM synthesis ( $y$-axis) for the synthetic $\mathrm{C} 1$ cluster without radio halo. Distribution histograms, along with mean and rms, are also given. Grey crosses indicate the contribution of background sources.

effects clearly also take place in the case of clusters without radio haloes.

To quantify the precision with which data trace the intrinsic RM, it is useful to compute the average difference in per cent between the results of RM synthesis $\sigma_{\mathrm{RM}}^{\text {out }}$ and the intrinsic $\sigma_{\mathrm{RM}}^{\text {in }}$ :

$\langle\Delta\rangle=\frac{1}{N} \sum_{i}^{N} \frac{\left|\sigma_{\mathrm{RM}}^{\text {out }}-\sigma_{\mathrm{RM}}^{\text {in }}\right|}{\sigma_{\mathrm{RM}}^{\text {in }}}$,

considering the $N$ data points available in each annulus. The $\langle\Delta\rangle$ values are shown on the right of the panels and shadow regions are traced at 1,2 and $3 \times\langle\Delta\rangle$.

Simulated data show mean differences of $\sim 8-15$ percent with respect to the $\sigma_{\mathrm{RM}}^{\text {in }}$ values, where the smallest values correspond to clusters without radio haloes. For synthetic data, the differences are $\sim 18-51$ per cent with respect to the input values, where the largest values occur for clusters without radio haloes.

From these results, it is clear that the $\sigma_{\mathrm{RM}}$ profile retrieved with the procedure proposed here is a good diagnostic tool for cluster magnetic field measurements within $\sim 20-30$ per cent when a diffuse source is hosted by the cluster independently from the scenario which triggers its emission and within $\sim 50$ percent for clusters without radio haloes.

\subsection{The RM structure function}

The RM structure function is defined as

$S(\mathrm{~d} r)=\left\langle[R M(r)-R M(r+\mathrm{d} r)]^{2}\right\rangle_{(x, y)}$,

where $r$ is the distance in $\mathrm{kpc}, S(\mathrm{~d} r)$ is in $\mathrm{rad}^{2} / \mathrm{m}^{4}$ and $\langle\ldots\rangle_{(x, y)}$ means that the average is taken over all positions $(x, y)$ in the RM image.

The structure function is computed in a circle of $1 \mathrm{Mpc}$ radius, centred on the cluster centres, with logarithmic sampling from $\sim 10 \mathrm{kpc}$ up to the maximum scale of $\sim 2 \mathrm{Mpc}$. By propagating the $\mathrm{RM}$ errors, the uncertainty associated with the structure function measurements becomes

$\Delta S(\mathrm{~d} r)=\frac{2 \sqrt{2 S(\mathrm{~d} r)}}{\sqrt{N}} \Delta \phi(l)$,

where $\Delta \phi(l)$ has been defined in equation (11) and $N$ is the number of measurements for each value of $S(\mathrm{~d} r)$.

Fig. 15 shows the resulting structure functions for the $\mathrm{C} 1$ (top) and $\mathrm{C} 2$ (bottom) clusters without the radio halo signal. The left panels refer to the simulated data, while on the right the results obtained considering the synthetic data are plotted. In each panel, magenta solid lines represent the structure function computed from the intrinsic RM images (Fig. 3) and points and dotted red lines refer to the structure function computed from the output Faraday depths (right images of Figs 9 and 10). The error bars are comparable with the size of the dots. In the same way, Fig. 16 shows the resulting structure functions for clusters with radio haloes simulated assuming the equipartition condition. The results concerning radio haloes obtained with coupling between thermal and non-thermal particle energy densities are similar to those obtained for radio haloes in equipartition.

The RM structure functions present an increase up to scales $\sim 100-200 \mathrm{kpc}$, followed by a flat behaviour with a very smooth increase and a decrease at $\sim 1 \mathrm{Mpc}$, due to poor sampling on large scales.

Going from simulated to synthetic data, it is possible to observe an irregular trend of the RM structure function traced by clusters without radio haloes. As for the $\sigma_{\mathrm{RM}}$ profile, this could be related to beam depolarization and the presence of thermal noise. As a consequence, synthetic data sample a smaller portion of the cluster area with respect to simulated data. Indeed, when diffuse sources are considered, it is noticeable that the synthetic data follow the intrinsic RM structure function much more faithfully.

As for the $\sigma_{\mathrm{RM}}$ profile, mean differences $\langle\Delta\rangle$ between the data and the intrinsic RM structure function have been evaluated: they are shown in the bottom right corner of each panel. For clusters without radio haloes, the resulting values go from a minimum of $\sim 20$ percent in the case of the simulated data up to a maximum of $\sim 236$ per cent in the case of the synthetic $\mathrm{C} 1$ cluster. In the case of clusters with radio haloes, the mean discrepancies with respect to the intrinsic value are $\sim 30$ per cent for simulated data, while for synthetic data they are of the order of $\sim 35-80$ per cent.

Shadow regions have been drawn for reference at 1,2 and $3 \times$ $\langle\Delta\rangle$. We note that the intrinsic profile lies within the $2 \times\langle\Delta\rangle$ region of synthetic data with haloes, while the profile for clusters without radio haloes is within the $3 \times\langle\Delta\rangle$ region up to a distance of $\sim 1 \mathrm{Mpc}$. The $\langle\Delta\rangle$ values clearly show that a good and homogeneous sampling of the RM is necessary to trace the intrinsic RM structure function.

\section{CONCLUSIONS}

In this work, the RM-synthesis technique has been applied to synthetic SKA1-MID radio images of a pair of galaxy clusters at $1.4 \mathrm{GHz}$ with a resolution of $10 \mathrm{arcsec}$ and a thermal noise of $0.1 \mu \mathrm{Jy}_{\text {beam }}{ }^{-1}$.

The ICM properties have been produced from cosmological MHD simulations and analysed. Using the FARADAY software package, the total intensity and linearly polarized emission of the clusters were modelled. This emission is related to a population of cluster, background and foreground radio galaxies and star-forming galaxies. Two models have been considered to simulate diffuse synchrotron sources at the centre of the clusters: one assumes equipartition between the relativistic particle energy density and 

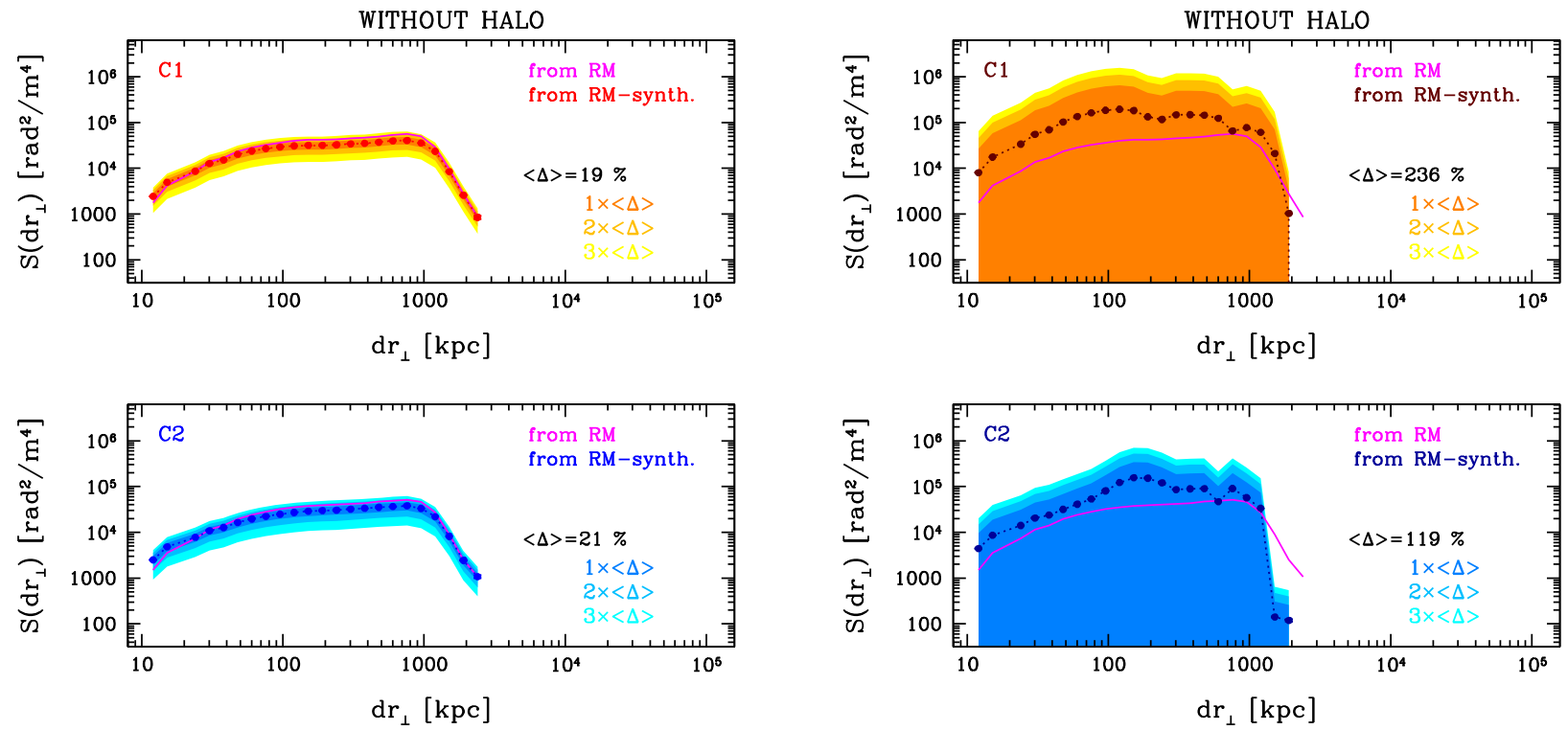

Figure 15. RM structure function of clusters without radio haloes for the $\mathrm{C} 1$ (top) and $\mathrm{C} 2$ clusters (bottom), respectively. On the left are results obtained for simulated data, on the right results for synthetic data. In each panel, magenta solid lines represent the structure function computed from the intrinsic RM images (Figs 3, and 10) and points and dotted red lines refer to the structure function computed from the output Faraday depth (right images of Figs 9 and 10). $\langle\Delta\rangle$ indicates the average difference in absolute value between the results of RM synthesis and the intrinsic RM (magenta line).
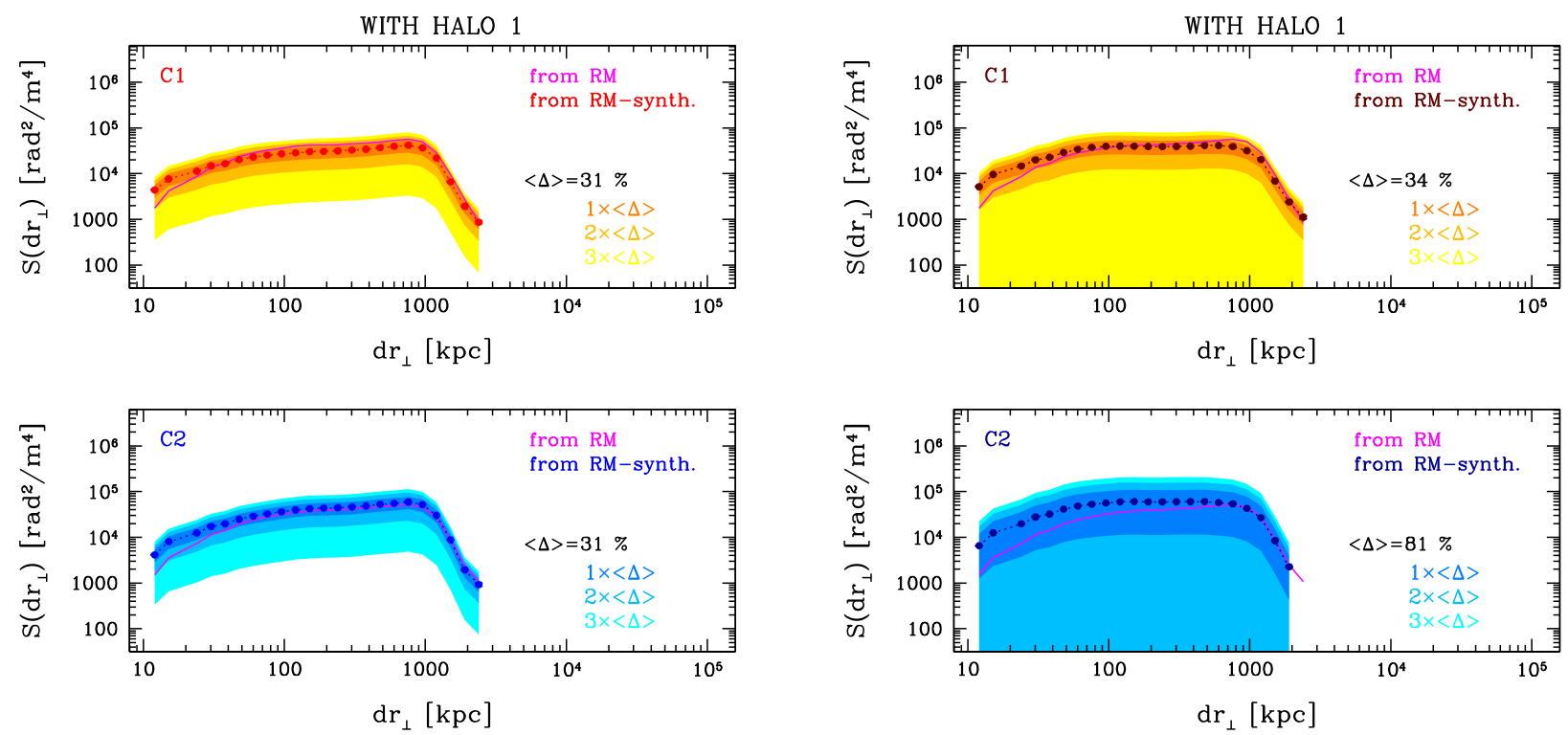

Figure 16. RM structure function of clusters with radio haloes in equipartition (HALO 1). On the left, the results obtained for simulated data are shown, while on the right are the results for synthetic data for the $\mathrm{C} 1$ (top) and $\mathrm{C} 2$ clusters (bottom), respectively. In each panel, magenta solid lines represent the structure function computed from the intrinsic RM images (Figs 3 and 10) and points and dotted red lines refer to the structure function computed from the output Faraday depth (central images of Figs 9 and 10). $\langle\Delta\rangle$ indicates the average difference in absolute value between the results of RM synthesis and the intrinsic RM (magenta line).

magnetic field energy density on one side and the other forces the relativistic particle energy density to be a fraction of 0.3 per cent of the thermal particle energy density.

RM synthesis has been applied in order to determine the polarized intensity of the sources and the cluster RM. The results were compared with the input information for both clusters and three simulated cases (clusters without radio haloes, clusters with radio haloes in equipartition, clusters with radio haloes and simulated coupling between thermal and non-thermal particles), considering either the polarized intensity, the $\sigma_{\mathrm{RM}}$ profile or the RM structure function.

The results can be summarized as follows.

(i) The two models assumed for radio haloes produce emission in both total intensity and polarization with different extension and morphology. Indeed, radio haloes in equipartition show more 
pronounced filamentary emission, which extends at large distances from the cluster centre with a discontinuous arrangement of bright and faint filaments, while radio haloes simulated assuming coupling between non-thermal and thermal plasma energy density show smoother emission, rapidly decreasing going to the outskirts. These differences are useful to assess the nature of relativistic particles in radio haloes, which will become possible with the advent of next-generation radio telescopes such as the SKA.

(ii) Even if their characteristics are different, the two kinds of cluster radio haloes do not show significant discrepancies in RM images, $\sigma_{\mathrm{RM}}$ profiles and RM structure functions.

(iii) The comparison between the linearly polarized signal at $\mathrm{RM}=0$ and the signal resulting from the application of RM synthesis demonstrate the efficiency of this technique in recovering polarization, especially at the centre of the clusters, where high values of RM can significantly depolarize signals with a percentage of $\sim 40-60$ per cent.

(iv) Synthetic data trace the instrinsic $\sigma_{\mathrm{RM}}$ profiles, within a percentage of $\sim 20-30$ per cent for clusters hosting radio haloes and $\sim 50$ per cent for clusters without radio haloes. A good sampling of the cluster area is of fundamental importance to constrain the cluster $\sigma_{\mathrm{RM}}$ and the presence of diffuse polarized radio sources can play a key role in the determination of cluster RM properties.

(v) A similar conclusion can be drawn for the RM structure function. Synthetic data of clusters without radio haloes reconstruct an irregular trend of this function and present average differences of between 120 and 240 per cent with respect to the intrinsic values. On the other hand, the presence of a radio halo, independently from the model assumed to reproduce its emission, can guarantee a better estimation of the intrinsic RM structure function, with discrepancies of the order of 30-80 per cent.

It is worth recalling that the results obtained in this work are model-dependent. Discrete radio sources have been modelled with very recent radio luminosity functions and on the basis of what is known about their polarization properties (see Loi et al. 2019 for more details). Despite the high expectations of RM measurements from polarized extragalactic sources with the SKA, this study shows that, with a resolution of $10 \operatorname{arcsec}$ and thermal noise in $Q$ and $U$ data of $0.1 \mu \mathrm{Jy}_{\text {beam }}{ }^{-1}$, the majority of background sources do not emerge from the noise and cannot therefore give us a RM value.

With SKA1-MID, several surveys have been proposed to be conducted at resolutions of 0.5 and 2 arcsec. Polarized images at such resolutions are less affected by beam depolarization and could drive different results, with intrinsic $\sigma_{R \mathrm{M}}$ profiles and the RM structure function traced in a better way by synthetic data. To investigate this point, MHD cubes of higher spatial resolution are needed; this will be the subject of future work.

To conclude, it is important to notice the key role that diffuse radio sources can play in determination of the RM and therefore intracluster magnetic field properties.

\section{ACKNOWLEDGEMENTS}

We gratefully acknowledge the anonymous referee for useful comments and suggestions. We also thank E. Bonnasieux, who carefully read the manuscript, helping us to improve it. FL and $\mathrm{AB}$ acknowledge financial support from the Italian Minister for Research and Education (MIUR), project FARE SMS, code R16RMPN87T. AB acknowledges financial support from the ERCStg DRANOEL, no 714245. IP acknowledges funding from the INAF PRIN-SKA 2017 project 1.05.01.88.04 (FORECaST). IP,
FL and MM acknowledge support from INAF under the MAIN STREAM PRIN project 'SAUROS'. The TRG computer cluster was funded by the Autonomous Region of Sardinia (RAS) using resources from the Regional Law 7 August 2007 n. 7 (year 2015) 'Highly qualified human capital', in the context of the research project CRP 18 'General relativity tests with the Sardinia Radio Telescope' (P.I. of the project: Dr. Marta Burgay).

\section{REFERENCES}

Bonafede A. et al., 2009, A\&A, 503, 707

Bonafede A., Feretti L., Murgia M., Govoni F., Giovannini G., Dallacasa D., Dolag K., Taylor G. B., 2010, A\&A, 513, A30

Brentjens M. A., de Bruyn A. G., 2005, A\&A, 441, 1217

Brunetti G., Zimmer S., Zandanel F., 2017, MNRAS, 472, 1506

Burn B. J., 1966, MNRAS, 133, 67

Cavaliere A., Fusco-Femiano R., 1976, A\&A, 49, 137

Clarke T. E., 2004, J. Korean Astron. Soc., 37, 337

Collins D. C., Xu H., Norman M. L., Li H., Li S., 2010, ApJS, 186, 308

Condon J. J., Cotton W. D., Greisen E. W., Yin Q. F., Perley R. A., Taylor G. B., Broderick J. J., 1998, AJ, 115, 1693

Dolag K., Schindler S., Govoni F., Feretti L., 2001, A\&A, 378, 777

Donnert J., Vazza F., Brüggen M., ZuHone J., 2018, Space Sci. Rev., 214 122

Enßlin T. A., Vogt C., 2003, A\&A, 401, 835

Fanaroff B. L., Riley J. M., 1974, MNRAS, 167, 31P

Felten J. E., 1996, in Virginia T., Andreas R., eds, ASP Conf. Ser. Vol. 88, Clusters, Lensing, and the Future of the Universe. Astron. Soc. Pac., San Francisco, p. 271

Feretti L., Giovannini G., Govoni F., Murgia M., 2012, A\&AR, 20, 54

Ferrari C., Govoni F., Schindler S., Bykov A. M., Rephaeli Y., 2008, Space Sci. Rev., 134, 93

Giovannini G., Vacca V., Girardi M., Feretti L., Govoni F., Murgia M., 2013, MNRAS, 435, 518

Girardi M. et al., 2016, MNRAS, 456, 2829

Govoni F., Feretti L., 2004, Int. J. Mod. Phys. D, 13, 1549

Govoni F., Murgia M., Feretti L., Giovannini G., Dallacasa D., Taylor G. B., 2005, A\&A, 430, L5

Govoni F., Murgia M., Feretti L., Giovannini G., Dolag K., Taylor G. B., 2006, A\&A, 460, 425

Govoni F., Ferrari C., Feretti L., Vacca V., Murgia M., Giovannini G., Perley R., Benoist C., 2012, A\&A, 545, A74

Govoni F., Murgia M., Xu H., Li H., Norman M. L., Feretti L., Giovannini G., Vacca V. 2013, A\&A, 554, A102

Govoni F. et al., 2017, A\&A, 603, A122

Govoni F. et al., 2019, Science, 364, 981

Guidetti D., Murgia M., Govoni F., Parma P., Gregorini L., de Ruiter H. R., Cameron R. A., Fanti R., 2008, A\&A, 483, 699

Guidetti D., Laing R. A., Murgia M., Govoni F., Gregorini L., Parma P., 2010, A\&A, 514, A50

Laing R. A., Bridle A. H., Parma P., Murgia M., 2008, MNRAS, 391, 521

Lawler J. M., Dennison B., 1982, ApJ, 252, 81

Lin Y.-T., Mohr J. J., 2007, ApJS, 170, 71

Loi F. et al., 2017, MNRAS, 472, 3605

Loi F., Murgia M., Govoni F., Vacca V., Prandoni I., Bonafede L., Feretti L., 2019, MNRAS, 485, 5285

Martinez Aviles G. et al., 2016, A\&A, 595, A116

Murgia M., Govoni F., Feretti L., Giovannini G., Dallacasa D., Fanti R., Taylor G. B., Dolag K., 2004, A\&A, 424, 429

Murgia M., Govoni F., Feretti L., Giovannini G., 2010, A\&A, 509, A86

Parekh V., Dwarakanath K. S., Kale R., Intema H., 2017, MNRAS, 464, 2752

Shakouri S., Johnston-Hollitt M., Pratt G. W., 2016, MNRAS, 459, 2525

Sotomayor-Beltran C. et al., 2013, A\&A, 552, A58

Taylor A. R., Stil J. M., Sunstrum C., 2009, ApJ, 702, 1230 
Vacca V., Murgia M., Govoni F., Feretti L., Giovannini G., Orrù E., Bonafede A., 2010, A\&A, 514, A71

Vacca V., Murgia M., Govoni F., Feretti L., Giovannini G., Perley R. A., Taylor G. B., 2012, A\&A, 540, A38

Vacca V. et al., 2018, MNRAS, 479, 776

van Weeren R. J., Bonafede A., Ebeling H., Edge A. C., Brüggen M. , Giovannini G., Hoeft M., Röttgering H. J. A., 2012, MNRAS, 425, L36 van Weeren R. J., de Gasperin F., Akamatsu H., Brüggen M., Feretti L., Kang H., Stroe A., Zandanel F., de Gasperin F., Akamatsu H., Brüggen M., Feretti L., Kang H., Stroe A., Zandanel F., 2019, Space Sci. Rev., 215,16

Xu H. et al., 2012, ApJ, 759, 40

\section{APPENDIX A: SINGLE-SCALE MAGNETIC FIELD MODELS COMPARED WITH DATA}

The $\sigma_{\mathrm{RM}}$ profile and the RM structure function are useful to determine the power spectrum of the intracluster magnetic field and therefore its strength and structure. To do so, it is necessary to apply advanced computational techniques, which, starting from modelling of the thermal plasma and intracluster magnetic field, produce synthetic images of the RM and, by comparing these images with observations, can determine the magnetic field characteristics. This interpretative part is beyond the scope of this work and would require a very long computational time (see e.g. Govoni et al. 2017). However, it is possible to give a rough estimate of the intracluster magnetic field strength in a simple way.

A magnetic field tangled on a single scale $\Lambda_{\mathrm{C}}$ randomly oriented from cell to cell results in a Gaussian-like RM distribution with zero mean and dispersion $\sigma_{\mathrm{RM}}$ (Lawler \& Dennison 1982; Felten 1996). Assuming a $\beta$ model for the thermal density (see equation 4 ) and a magnetic field strength radial profile that is a function of the thermal gas density,

$B=B_{0}\left(\frac{n}{n_{0}}\right)^{\eta}$,

where $\eta$ and $B_{0}$ are the exponential factor and the central value of the magnetic field, $\sigma_{\mathrm{RM}}$ as a function of the projected distance $r$ is (Dolag et al. 2001)

$$
\begin{array}{r}
\sigma_{\mathrm{RM}}(r)=K B_{0} \Lambda_{\mathrm{c}}^{0.5} n_{0} r_{\mathrm{c}}^{1 / 2} \frac{1}{\left(1+\frac{r^{2}}{r_{\mathrm{c}}^{2}}\right)^{[6 \beta(1+\eta)-1] / 4}} \\
\times \sqrt{\frac{\Gamma\left[3 \beta(1+\eta)-\frac{1}{2}\right]}{\Gamma[3 \beta(1+\eta)]}} .
\end{array}
$$

The factor $K$ is a constant, which depends on the integral path: in particular, $K=624$ for background sources and 441 for sources lying in the middle of the cluster.

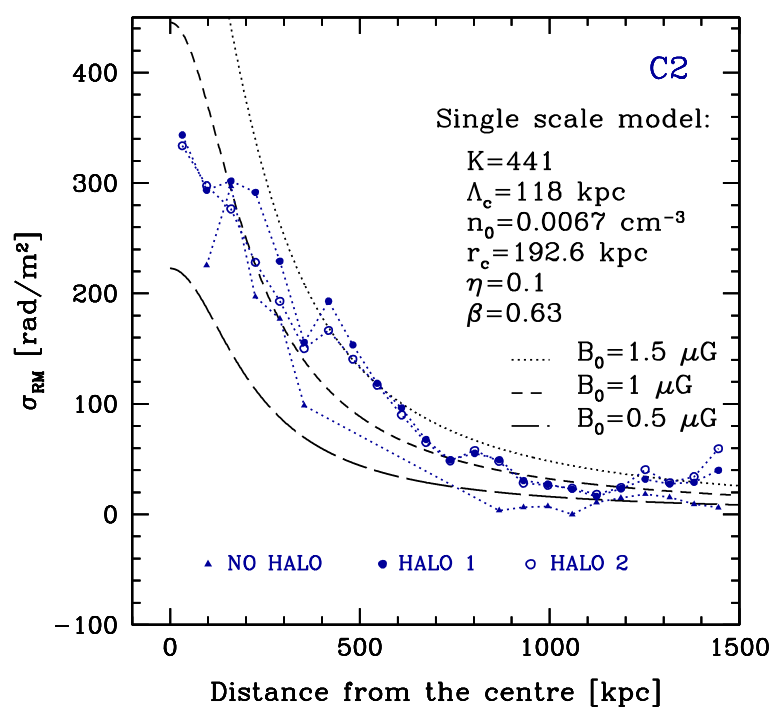

Figure A1. Comparison of the synthetic $\sigma_{\mathrm{RM}}$ profile of the $\mathrm{C} 2$ cluster without a radio halo (triangles), with a radio halo in equipartition (full circles) and coupled with thermal plasma (open circles) with the singlescale magnetic field model, assuming $B_{0}=0.5,1,1.5 \mu \mathrm{G}$.

Fig. A1 shows the comparison between the $\sigma_{\mathrm{RM}}$ synthetic data of the $\mathrm{C} 2$ cluster and the single-scale model. To trace the profile of equation (A2), the values reported in Section 2 have been used, namely $\Lambda_{\mathrm{c}}=118 \mathrm{kpc}, n_{0}=0.0067 \mathrm{~cm}^{-3}, r_{\mathrm{c}}=192.6 \mathrm{kpc}, \beta=$ 0.63 . The fit of the $\log \left(B / B_{0}\right)-\log \left(n / n_{0}\right)$ profiles between 100 and $500 \mathrm{kpc}$ yields a power law with index $\eta=0.1$. Assuming $K=441$, three profiles are plotted considering $B_{0}=0.5,1,1.5 \mu \mathrm{G}$. In all three cases, the data are better described with the single-scale model with $B_{0}=1 \mu \mathrm{G}$ and indeed the $\mathrm{C} 2$ intracluster magnetic field has a central strength of $\sim 0.8-0.9 \mu \mathrm{G}$ (see Fig. 2).

This paper has been typeset from a $\mathrm{T}_{\mathrm{E}} \mathrm{X} / \mathrm{L} \mathrm{T}_{\mathrm{E}} \mathrm{X}$ file prepared by the author. 